\title{
Extensive Tonotopic Mapping across Auditory Cortex Is Recapitulated by Spectrally Directed Attention and Systematically Related to Cortical Myeloarchitecture
}

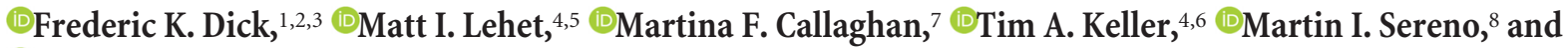 \\ (ㄴ)Lori L. Holt ${ }^{4,5}$ \\ ${ }^{1}$ Department of Psychological Sciences, Birkbeck College, University of London, London, WC1E 7HX, United Kingdom, ${ }^{2 B i r k b e c k / U n i v e r s i t y ~ o f ~ L o n d o n ~}$ \\ Centre for Neuroimaging, London, WC1H 0AP, United Kingdom, ${ }^{3}$ Department of Experimental Psychology, University College London, London, WC1H \\ 0AP, United Kingdom, ${ }^{4}$ Department of Psychology, Carnegie Mellon University, Pittsburgh, Pennsylvania 15213, ${ }^{5}$ Center for the Neural Basis of Cognition, \\ Carnegie Mellon University, Pittsburgh, Pennsylvania 15213, ${ }^{6}$ Scientific Imaging and Brain Research Center, Carnegie Mellon University, Pittsburgh, \\ Pennsylvania 15213, ${ }^{7}$ Wellcome Trust Center for Neuroimaging, Institute of Neurology, University College London, London, WC1N 3BG, United Kingdom, \\ and ${ }^{8}$ Department of Psychology, San Diego State University, San Diego, California San Diego, California 92182-4611
}

Auditory selective attention is vital in natural soundscapes. But it is unclear how attentional focus on the primary dimension of auditory representation-acoustic frequency — might modulate basic auditory functional topography during active listening. In contrast to visual selective attention, which is supported by motor-mediated optimization of input across saccades and pupil dilation, the primate auditory system has fewer means of differentially sampling the world. This makes spectrally-directed endogenous attention a particularly crucial aspect of auditory attention. Using a novel functional paradigm combined with quantitative MRI, we establish in male and female listeners that human frequency-band-selective attention drives activation in both myeloarchitectonically estimated auditory core, and across the majority of tonotopically mapped nonprimary auditory cortex. The attentionally driven best-frequency maps show strong concordance with sensory-driven maps in the same subjects across much of the temporal plane, with poor concordance in areas outside traditional auditory cortex. There is significantly greater activation across most of auditory cortex when best frequency is attended, versus ignored; the same regions do not show this enhancement when attending to the least-preferred frequency band. Finally, the results demonstrate that there is spatial correspondence between the degree of myelination and the strength of the tonotopic signal across a number of regions in auditory cortex. Strong frequency preferences across tonotopically mapped auditory cortex spatially correlate with $\mathrm{R}_{1}$-estimated myeloarchitecture, indicating shared functional and anatomical organization that may underlie intrinsic auditory regionalization.

Key words: attention; auditory; cortical mapping

Significance Statement

Perception is an active process, especially sensitive to attentional state. Listeners direct auditory attention to track a violin's melody within an ensemble performance, or to follow a voice in a crowded cafe. Although diverse pathologies reduce quality of life by impacting such spectrally directed auditory attention, its neurobiological bases are unclear. We demonstrate that human primary and nonprimary auditory cortical activation is modulated by spectrally directed attention in a manner that recapitulates its tonotopic sensory organization. Further, the graded activation profiles evoked by single-frequency bands are correlated with attentionally driven activation when these bands are presented in complex soundscapes. Finally, we observe a strong concordance in the degree of cortical myelination and the strength of tonotopic activation across several auditory cortical regions.

\section{Introduction}

Listeners shift attention across multiple simultaneously present acoustic dimensions to home in on those that are diagnostic in

Received May 24, 2017; revised 0ct. 4, 2017; accepted 0ct. 6, 2017.

Author contributions: F.K.D. and L.L.H. designed research; F.K.D., M.I.L., T.A.K., and L.L.H. performed research; M.F.C. and M.I.S. contributed unpublished reagents/analytic tools; F.K.D., M.I.L., and L.L.H. analyzed data; F.K.D., M.F.C., M.I.S., and L.L.H. wrote the paper. guiding behavior (Idemaru and Holt, 2011; Herrmann et al., 2013; Shamma and Fritz, 2014). In nonhuman animal studies, task-based spectral attention adaptively modulates auditory neu- 
rons' spectrotemporal response fields (Fritz et al., 2010). Human neuroimaging results reveal that attention to streams of highversus low-frequency acoustic input can modulate activity in tonotopically defined regions (Paltoglou et al., 2009), as can imagery of higher versus lower frequencies (Oh et al., 2013). In and directly around Heschl's gyrus, there are strong frequency bandspecific attentional effects to high and low pure-tone streams presented to opposite ears (Da Costa et al., 2013) and a shared topography of sensory and attentionally driven responses (Riecke et al., 2017). These results establish that endogenous attention directed across acoustic frequency, the primary axis of auditory representation, can modulate human cortical activity in a tonotopic manner around Heschl's gyrus. But there remain important unanswered questions about the neurobiological basis of human spectrally directed attention.

First, does the topography of attention to different frequency bands recapitulate tonotopic organization in human primary auditory cortex? Nonhuman animal physiology establishes spectrally directed attention in myeloarchitectonically and cytoarchitectonically defined primary areas in "auditory core" (Fritz et al., 2007b; Shamma and Fritz, 2014). However, although two recent neuroimaging studies have shown strong similarities between stimulus-driven and attentionally driven tonotopic organization in and directly around Heschl's gyrus (Da Costa et al., 2013; Riecke et al., 2017), it has not yet been possible to unambiguously localize this effect to human auditory core. Here, we use highresolution quantitative MRI (Pierpaoli, 2010) to estimate myeloarchitectonically defined auditory core, and demonstrate that spectrally directed attention modulates its activation in a tonotopically organized manner.

Second, is attentionally driven tonotopic organization present outside of auditory core? In humans, Riecke et al. (2017) found no significant evidence for tonotopically organized effects of spectral attention outside of early auditory areas but did show that the information content of nonprimary cortical frequency representations was sufficient for above-chance decoding of listeners' frequency-selective attentional focus. The lack of attentionally driven tonotopic maps contrasts with the finding that most nonprimary cortical visual areas exhibit strong retinotopically specific attentional effects (Saygin and Sereno, 2008). Using intensive data collection ( $>7000$ functional volumes per subject), we present evidence for widespread, tonotopically organized modulation by spectral attention across much of auditory cortex, with individual differences in individual participants' tonotopic maps reproduced in attentionally driven maps.

Third, what is the effect of frequency-selective attention being directed to a voxel's nonpreferred frequency band? Detailed fMRI studies of stimulus-driven frequency response functions (Schönwiesner and Zatorre, 2009; Moerel et al., 2013) have shown

Antoine Lutti and Nikolaus Weiskopf for physics expertise and generosity with porting the multiparameter mapping protocol to the SIBR Verio; the developers of FSL, AFNI, and FreeSurfer for scientific work and software; and Marlene Behrmann, Jenny Bizley, Maria Chait, Tim Griffiths, Jen Linden, Catherine Perrodin, Chris Petkov, Lars Riecke, Sam Schwarzkopf, Jeremy Skipper, Ediz Sohoglu, Adam Tierney, and three anonymous reviewers of a previous version of the manuscript for extremely useful suggestions and feedback.

The authors declare no competing financial interests.

Correspondence should be addressed to either of the following: Dr. Frederic K. Dick, Birkbeck/University of London Centre for Neurolmaging, 26 Bedford Way, London, WC1H 0AP, United Kingdom, E-mail: f.dick@bbk.ac.uk; or Dr. Lori L. Holt, Department of Psychology, Baker Hall, Carnegie Mellon University, 5000 Forbes Avenue, Pittsburgh, PA 15213, E-mail: loriholt@cmu.edu.

DOI:10.1523/JNEUROSCI.1436-17.2017 Copyright $\odot 2017$ Dick et al.

This is an open-access article distributed under the terms of the Creative Commons Attribution License Creative Commons Attribution 4.0 International, which permits unrestricted use, distribution and reproduction in any medium provided that the original work is properly attributed. graded and multipeaked frequency responses across human auditory cortex. However, it is unclear whether these more complex patterns are recapitulated by attention to a given frequency band. In the context of three distinct frequency bands, Riecke et al. (2017) found that attentional filters appeared to be bandpass in and around Heschl's gyrus. Here, using a five-frequencyband paradigm, we establish that graded response profiles evoked by single-frequency bands are strongly associated with attentionally driven response profiles to those frequencies across much of auditory cortex. We also show that a systematic topography of "dis-preferred" frequency can be driven by attention, and establish the regionalization of spectral attentional effects relative to prior studies of cross-modal auditory attention (Petkov et al., 2004).

Finally, is there spatial correspondence between auditory cortical anatomy, as measured by the local change in $\mathrm{R}_{1}$-estimated myelination, and fMRI-assessed strength of relative frequency selectivity? Postmortem Gallyas staining to establish human myeloarchitecture reveals considerable variability in auditory cortical myelination that is associated with MRI signal change in the same brain (Wallace et al., 2016). Likewise, variation in cortical myelination estimated using T1-weighted/T2-weighted ratio approaches also appears to correspond spatially with some functional variation in the superior temporal lobe (Glasser et al., 2016). Here, we demonstrate that there is spatial concordance between the degree of myelination and the amplitude of the frequency-selective tonotopic signal across several regions in auditory cortex.

\section{Materials and Methods}

Experiment overview. We used a novel paradigm in which listeners direct attention to a series of four-tone "mini-sequences" that fall within one of five possible spectral bands, without any spatial cues. The task is to monitor for temporally adjacent mini-sequence repeats within the attended band. Because this places a very high demand on encoding and integrating spectral sequences within a delimited frequency range, we expect it to be especially effective in evoking strong responses in nonprimary auditory cortical areas. The goal is to address where specifically in the auditory system spectral gain from attention is evident, and akin to long-standing work in vision (Kastner and Ungerleider, 2000), to delineate the topographic maps across which attentional modulation is apparent.

The target mini-sequences were embedded in either an informationally sparse or informationally dense acoustic scene (Fig. 1). Streams of four-tone mini-sequences were presented in either a single band ("tonotopy," Fig. 1A), or accompanied by mini-sequences in a "distractor" frequency band, the center frequency of which varied in the frequency distance from the attended band across blocks (attention-tonotopy, [attn-tono]; Fig. 1B). A verbal cue directed listeners' attention to a specific frequency band, within which listeners monitored four-tone minisequences for repeats; the distractor band in attn-tono blocks also contained repeats. Using a discretized version of a phase-encoded fMRI design (Sereno et al., 1995; Rao and Talavage, 2005; Schwarzkopf et al., 2011; Herdener et al., 2013; Langers et al., 2014), the cued frequency band stepped up or down in orderly steps across the acoustic spectrum across a 64 s cycle (Figure $1 C$ ). Phase-encoded tonotopic designs benefit from the power and robustness of the "traveling wave" method for topographic cortical mapping of smoothly varying representations (Engel, 2012); the discretized (blocked) version we use here allows use of the verbal cue and has the advantage of being able to be analyzed using both Fourier and regression approaches. This allowed us to include an additional, "randomized" attn-tono condition that contributed both as a control condition in Fourier analyses and also as an additional attn-tono run in regression analyses (Fig. 1D). The tone stimuli from this condition were identical to the "stepped" attn-tono condition, but the order of the verbal cues directing listeners' attention to a specific frequency band was scrambled in their assignment to blocks. This preserved the acoustics across conditions but eliminated the consistent "stepping" of attention 


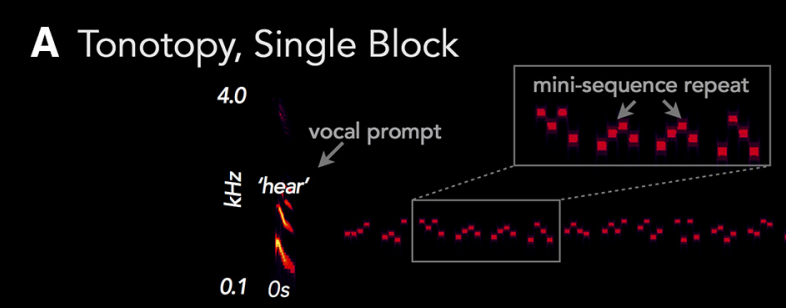

\section{B Attention-Tonotopy, Single Block}

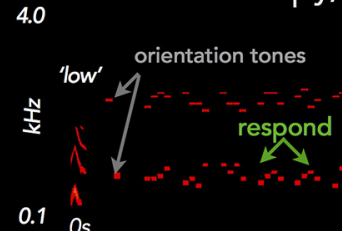

$12.8 s$

0.1 Os

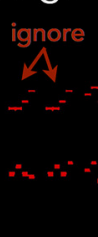

D Attention-tonotopy, Randomized Cycle

4.0
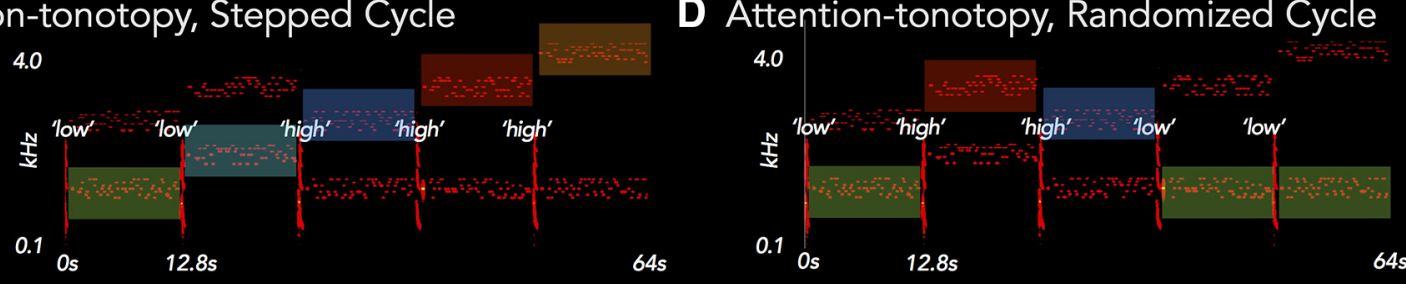

\begin{tabular}{|c|c|c|c|c|c|}
\hline Attended Band & $300 \mathrm{~Hz}$ & $566 \mathrm{~Hz}$ & $1068 \mathrm{~Hz}$ & $2016 \mathrm{~Hz}$ & $3805 \mathrm{~Hz}$ \\
\hline
\end{tabular}

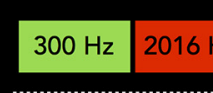

$\begin{array}{llllll}0 s & 12.8 \mathrm{~s} & 25.6 \mathrm{~s} & 38.4 \mathrm{~s} & 51.2 \mathrm{~s} & 64 \mathrm{~s}\end{array}$

\section{E Fourier Analyses \\ Attention-tonotopy, Stepped consistent relationship attention $=>$ phase lag}
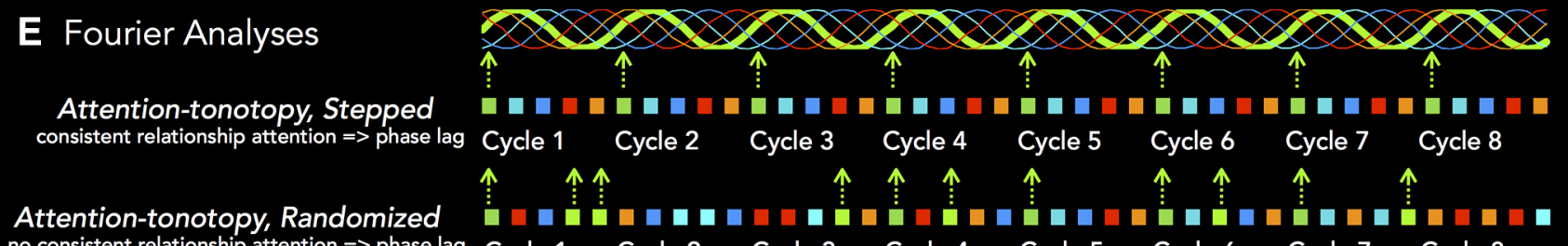

no consistent relationship attention $\Rightarrow$ phase la

Cycle 1

Cycle 2

Cycle 3

Cycle 4

Cycle 5

Cycle 6

Cycle 7

Cycle 8

$\uparrow \uparrow \uparrow \uparrow$

$\hat{1}$

$\begin{array}{llllll}\text { Cycle } 3 & \text { Cycle } 4 & \text { Cycle } 5 & \text { Cycle } 6 & \text { Cycle } 7 & \text { Cycle } 8\end{array}$

\section{F Fourier Surface Map}

Each voxel is painted with color corresponding to the stimulus phase lag with highest BOLD signal. Color saturation reflects BOLD amplitude.
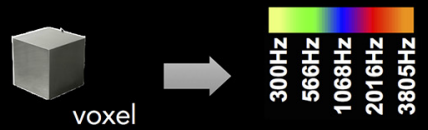

$2.8 s$ 
stepping down in frequency and averaged them with runs stepping up in frequency (Sereno et al., 1995; Talavage et al., 2004; Dick et al., 2012; Ahveninen et al., 2016). Cross-subject averaging of phase-encoded mapping data was performed using a method described previously (Hagler et al., 2007) in which the real and imaginary components of the signal with respect to the stepped cycle were sampled to the cortical surface and then averaged across subjects, preserving any phase information that was coherent over subjects.

Using previously established methods (Dick et al., 2012; Sereno et al., 2013; Lutti et al., 2014; see also Glasser et al., 2016), we used highresolution quantitative multiparameter mapping to generate maps of estimated cortical myelination based on longitudinal relaxation times (quantitative $\mathrm{T}_{1}$ ). Recent work by multiple laboratories supports the hypothesis that $\mathrm{T}_{1}$ relaxation is reliably associated with quantitative differences in myelination in white matter and cortex (Sereno et al., 2013; Stüber et al., 2014; Dinse et al., 2015; Callaghan et al., 2015; Tardif et al., 2015, 2016; Turner, 2015). Here, we calculated each subject's $\mathrm{R}_{1}\left(1 / \mathrm{T}_{1}\right)$ values, where the greater the $R_{1}$, the higher the inferred myelin content. These $R_{1}$ values were resampled onto his or her surface at a cortical depth fraction of 0.5 and also averaged across individuals using sulcus-aligned cortical-surface-based procedures (see below for further details).

Participants. Eight adults (aged 23-45 years, mean 28 years; 6 female) participated; none reported a history of neurological disease or communication disorders. All had some childhood and/or adult musical training (one had a music degree) and had previous experience with longer scanning sessions. While musical training seemed to facilitate learning the experimental task, subsequent behavioral studies in the laboratory have shown that musically naive subjects can also attain excellent performance with similar levels of training on this and even more demanding related tasks.

Stimuli and design. Stimuli were created using custom code in MATLAB version 2015a (The MathWorks) and SoX version 14.4.2 (www.sourceforge.net). The basic stimulus unit was a four-tone minisequence (140 ms sine-wave tones, including $10 \mathrm{~ms}$ linear amplitude ramp), with each tone drawn with replacement from a seven-semitone, band-delimited pool centered around one of five frequencies $(300,566$, 1068, 2016, and $3805 \mathrm{~Hz}$; Fig. 1A). Fourteen mini-sequences formed a block (mean intersequence silent interval $240 \mathrm{~ms}$, SD $10 \mathrm{~ms}$ ). Each block contained one to three mini-sequence repeats (1:2:1 ratio of 1,2 , and 3 repeats). When there was more than one repeat per block, mini-sequence repeat pairs were separated by at least one intervening mini-sequence. Each block began with a verbal prompt—-hear", "high", or "low"generated with a loudness-equalized Mac Victoria voice mean duration $506 \mathrm{~ms}$ (SD $36 \mathrm{~ms}$ ), padded with silence to $800 \mathrm{~ms}$ total duration. This prompt was followed by $800 \mathrm{~ms}$ silent gap (tonotopy) or tone-cue (attntono), then the 14 mini-sequences (11.2 s in total), for a total block duration of $12.8 \mathrm{~s}$.

The task was to detect mini-sequence repeats in the attended frequency band (i.e., a 1-back task). In the tonotopy condition, mini-sequences were confined to a single frequency band preceded by the neutral verbal prompt hear (Fig. 1A). In two of the four single-band tonotopy runs, block center frequency was stepped from low to high over a 64 s cycle with 8 cycles/run; step direction was reversed (high to low) for the other two runs. This is a "discrete" version of phase-encoded designs commonly used in visual, somatosensory, and auditory mapping studies (Engel et al., 1994; Sereno et al., 1995; Da Costa et al., 2011; Dick et al., 2012; Langers and van Dijk, 2012; Langers et al., 2014; Saenz and Langers, 2014).

The attn-tono condition had the exact mini-sequence patterns from the tonotopy blocks, but there also were simultaneous, competing minisequences in a distinct frequency band with a center frequency at least 14 semitones apart (Fig. 1B; 300 vs $1068 \mathrm{~Hz} ; 300$ vs $2016 \mathrm{~Hz} ; 300$ vs $3805 \mathrm{~Hz}$; 566 vs $2016 \mathrm{~Hz} ; 566$ vs $3805 \mathrm{~Hz} ; 1068$ vs $3805 \mathrm{~Hz}$; not all center frequencies were paired due to the 14 semitone constraint). The verbal prompt ("high" or "low") initiating each attn-tono block signaled participants to perform the 1-back task on either the higher or lower frequency band. Immediately after the verbal prompt, a randomly ordered pair of sinewave tones cued the center frequencies of the upcoming block $(140 \mathrm{~ms}$ tones, including $8 \mathrm{~ms}$ linear on/off amplitude ramp; $26 \mathrm{~ms}$ intertone silence, tone pair followed by $494 \mathrm{~ms}$ silence, total duration $800 \mathrm{~ms}$ ). Crucially, there were mini-sequence repeats even in the unattended band to assure that attention directed to the task was endogenously driven rather than being attracted by stimulus repetition effects (Barascud et al., 2016).

There were two attn-tono conditions: stepped and randomized. Analogous to single-band tonotopy runs, in stepped attn-tono runs, the verbally cued frequency band implicitly stepped up (2 runs) or down (2 runs) in frequency over a $64 \mathrm{~s}$ cycle (Fig. 1C). This cued iterative stepping through the frequency spectrum facilitates transfer of attention to each frequency band (as in traditional phase-encoded designs) and supports Fourier approaches to analysis (Fig. 1E). Each randomized attn-tono run was acoustically identical to a stepped run, but the verbal prompt was manipulated so that there was no systematic, stepped organization of attended mini-sequence center frequencies through the spectrum (Fig. $1 D)$. For this condition, frequency bands were cued at inconsistent phase lags within the 8 cycles/run, thereby phase-canceling any periodic attentional response; this is schematized in Figure $1 E$. This randomized-order control is important, as there is a small ( $\sim 1$ octave) overall shift in spectral mean over the course of an attn-tono stimulus cycle that is unavoidable due to the constraints on the pairing of frequency bands.

Each of the 12 9.6-min-long runs was composed of eight $64 \mathrm{~s}$ cycles plus $32 \mathrm{~s}$ silent periods at the beginning and end of each run to allow for calculation of baseline auditory activation (Klein et al., 2014).

Behavioral thresholds and training. Participants first underwent behavioral tests of monaural pure-tone thresholds and binaural thresholds for detecting mini-sequences in quiet and in acoustic noise generated by the MRI scanner running the multiband EPI sequence. This provided a basis for adjusting center frequency amplitudes to approximate equal loudness in scanner noise. Participants also trained on the mini-sequence detection task in quiet and in acoustic scanner noise across two sessions.

Imaging data acquisition. Structural and functional images were acquired on a 3 Tesla Siemens Verio wide-bore MRI scanner at the Scientific Imaging and Brain Research Center at Carnegie Mellon University using a phased array 32-channel head coil across three scan sessions on separate days. Stimulus presentation was under the control of a MacBookPro running PsychToolbox 3.0.12 in MATLAB, with audio output to an external AD/DA converter (Babyface, RME) connected to an amplifier (Pylepro) that delivered stimuli to participants in the scanner diotically over MRI-compatible earbuds (Sensimetrics S14). All stimuli were prefiltered to equalize sound stimuli according to the earbuds' frequency response. After participants were settled into the bore, sound volume was adjusted so that participants could comfortably hear all frequencies through scanner noise. Participants wore a fiber optic response glove (Current Designs) that communicated with a Brain Logics I/O device (Psychology Software Tools); participants used the glove to respond to minisequence repeats using the right index finger. During all functional scans, subjects closed their eyes to reduce the potential for stimulus-correlated eye movements.

In the initial scanning session ( $~ 50 \mathrm{~min})$, we acquired multiparameter mapping images for quantitative myelin mapping and structural identification of primary auditory cortex on an individual basis while participants watched a film. Proton density-weighted (PDw), T1weighted (T1w), and magnetization transfer (MTw) images were acquired using an in-house 3D FLASH pulse sequence (voxel size: $0.8 \times$ $0.8 \times 0.8 \mathrm{~mm}^{3}$, matrix $=320 \times 280 \times 208, \mathrm{TR}=25.0 \mathrm{~ms}$, bandwidth $488 \mathrm{~Hz} / \mathrm{px}$, excitation flip angle: $6^{\circ}(\mathrm{PDw} / \mathrm{MTw})$ or $21^{\circ}(\mathrm{T} 1 \mathrm{w})$, slab rotation $30^{\circ}$ ). To accelerate this high-resolution acquisition, a partial Fourier acquisition (6/8 coverage) was used in the inner phase-encoded direction (right, left) and parallel imaging was used along the outer phaseencoding direction (anteroposterior), reconstructed using the GRAPPA algorithm (acceleration factor 2, 18 integrated auto-calibration lines) as implemented on the scanner platform. Four gradient echoes were acquired for each contrast $(\mathrm{TE}=2.5,4.74,6.98,9.22 \mathrm{~ms}$ ) after each excitation pulse and averaged to improve signal-to-noise ratio (Helms et al., 2009). Each FLASH acquisition lasted 9 min 45 s. Quantitative $R_{1}(1 / T 1)$ maps were estimated from the PDw and T1w images according to the model developed by Helms et al. (2008), including a correction for RF transmit field inhomogeneities (Lutti et al., 2010) and imperfect spoiling (Preibisch and Deichmann, 2009). The transmit field map was calculated using a 3D EPI spin-echo/stimulated echo method (Lutti et al., 2010, 
2012) FOV $=256 \times 192 \times 192 \mathrm{~mm}$, matrix $=64 \times 64 \times 48, \mathrm{TE}=53.14$ $\mathrm{ms}, \mathrm{TM}=47.60 \mathrm{~ms}, \mathrm{TR}=500 \mathrm{~ms}$, bandwidth $=2298$, nominal $\alpha$ varying from $135^{\circ}$ to $65^{\circ}$ in steps of $5^{\circ}$, acquisition time $6 \mathrm{~min}$ ) and was corrected for off-resonance effects using a standard B0 field map (double gradient echo FLASH, $3 \times 3 \times 2 \mathrm{~mm}$ isotropic resolution, whole-brain coverage).

The final two scanning sessions acquired functional data for four runs each of the tonotopy, stepped attn-tono, and randomized attn-tono conditions. The runs were interleaved across conditions and designed to assess phase-encoded functional influences of selective attention across frequency (stepped attn-tono), the functional response to identical acoustics without systematic phase-encoded shifts of attention (randomized attntono), and functional responses to single-frequency bands identical to the attended bands in attn-tono, with phase-encoded steps through frequency and no distractor frequency bands (tonotopy). Across all functional runs, participants engaged in detecting repeats (1-back) of the four-tone minisequences. Run order was counterbalanced according to condition and whether the cycle involved steps up or down in frequency.

Functional images were acquired using a T2*-weighted EPI pulse sequence (44 oblique axial slices, in-plane resolution $3 \mathrm{~mm} \times 3 \mathrm{~mm}, 3 \mathrm{~mm}$ slice thickness, no gap, $\mathrm{TR}=1000 \mathrm{~ms}, \mathrm{TE}=41 \mathrm{~ms}$, flip angle $=61^{\circ}$, matrix size $=64 \times 64, \mathrm{FOV}=192 \mathrm{~mm}$ ). All EPI functional scans were performed using $4 \times$ multiband acceleration (Feinberg et al., 2010; Feinberg and Setsompop, 2013). There were 584 repetitions acquired per run, with the first 8 images discarded to allow for longitudinal magnetization to arrive at equilibrium. Runs were pseudo-randomly ordered across participants.

Image preprocessing: cortical surface creation, and mapping of $R_{1}$ values. Each subject's cortical surface was reconstructed from a contrast-optimized synthetic FLASH volume, created with mri_synthesize in Freesurfer from scaled and truncated versions of the T1 and proton-density volumes; another MPRAGE-like synthetic image was created for use with the automated Freesurfer Talairach procedure. Both volumes were conformed to $1 \mathrm{~mm}$ isotropic resolution and used in a customized reconstruction pipeline version. In particular, the subject's PD volume was used to deskull the synthetic FLASH image using a "shrink-wrap" technique (Dale and Sereno, 1993). After inspection for reconstruction quality, $\mathrm{R}_{1}$ values were resampled from 50\% cortical depth fraction to the subject's surface, and also morphed to the unit icosahedron for cross-subject curvature-aligned cortical-surface-based averaging (Fischl et al., 1999).

EPI processing. Each functional image from both sessions was aligned to a reference volume from the middle of the first run using AFNI's 3dvolreg; registration and motion correction goodness were hand-checked for each run. The reference volume was aligned to the subject's cortical surface using boundary-based registration in Freesurfer (Greve and Fischl, 2009), verified using manual blink comparison, and applied to the volume-aligned EPI data for resampling. EPI data were analyzed in native space without any spatial smoothing using both Fourier and general linear methods.

Experimental design and statistical analysis. As noted above, the fMRI experiment used a discrete version of a traditional phase-encoded design, such that both Fourier based and general linear model approaches could be used. Fourier analyses were performed in csurf (http://www.cogsci. ucsd.edu/ sereno/.tmp/dist/csurf) with individual and group analysis methods used as previously described (Sereno et al., 1995; Sereno and Huang, 2006; Hagler et al., 2007). Functional activation amplitude was estimated as the Fourier amplitude of the periodic BOLD signal (proportional to percent response) at the frequency of the stimulus cycle ( 8 repetitions per run). An $F$ statistic was calculated by comparing that amplitude to the average amplitude of other noise frequencies (Hagler et al., 2007). Periodic signal components with very low frequencies (due to slow head motion) and the second and third harmonic of the stimulus were excluded as neither signal nor noise (this is mathematically equivalent to first linearly regressing out these frequencies as nuisance variables before calculating significance). The phase of the signal, which corresponds to a particular point of the stimulus cycle, was then mapped to a color scale, and the amplitude of the signal at each vertex was mapped to color saturation (Gouraud shading within each face). Runs with downward frequency steps were time-reversed and averaged with upward-stepped scans to cancel fixed voxel-specific delays in the BOLD response.
Linear modeling was performed in FSL (Smith et al., 2004). For all runs, the motion-registered data were high-pass-filtered (100 s) and prewhitened; a hemodynamic model corresponding to each stimulated and attended (tonotopy condition) or attended (stepped, randomized attn-tono conditions) frequency band was created by convolving the $12.8 \mathrm{~s}$ block with a gamma function (lag $6 \mathrm{~s}$, SD 3s). In a separate multiple regression, the unattended (ignored) frequency band was modeled for both stepped and randomized attn-tono conditions. The verbal cue was also modeled; all models were temporally filtered before multiple regression. Coefficients from the first-level contrasts for each of the four runs were combined in a fixed-effects analysis for each condition; data from the stepped and random block conditions were also combined in an eight-run average.

Cross-subject averaging of phase-encoded mapping data was performed using the methodology developed by Hagler and Sereno (2006) in which the real and imaginary components of the signal with respect to the stimulus ramp are averaged across subjects, preserving any phase information consistent between subjects. This was performed by projecting each participant's phase-encoded map to the FreeSurfer spherical atlas using mri_surf2surf, performing 1 step of surface-based smoothing ( $<1 \mathrm{~mm}$ FWHM in 2D), averaging across subjects at each vertex, then painting back onto a single subject's surface for viewing. For the multiple regression analyses, the same sampling process was used to sample each subject's contrast parameter estimates for cross-subject averaging and $t$ tests.

Surface-based cluster exclusion was used to correct for multiple comparisons in the groupwise averages (surfclust and randsurfclust) (Hagler et al., 2006). The exclusion criterion (only surface clusters $>78 \mathrm{~mm}^{2}$ unless otherwise noted) was determined based on the minimum estimated cortical area from iterative random sampling of cluster sizes $(N=$ 10,000 iterations per hemisphere in randsurfclust) required to achieve a corrected $\alpha$ of $p<0.001$ for each hemisphere, based on an initial uncorrected $\alpha$ of vertexwise $p<0.01$.

As an alternative means of defining primary auditory cortex, we projected the Morosan et al. (2001) 3D raw probability maps provided in the AFNI (version 16.3.13) (Cox, 2012) to a FreeSurfer "fsaverage" brain registered to the Talairach target brain, resampled the data onto the cortical surface, and thresholded at $p>0.30$ to create region of interest (ROI) labels. The labels were $\sim 2 \mathrm{~mm}$ FWHM (five steps) surface-smoothed with manual removal of isolated marked vertices (due to "spillover" from the $3 \mathrm{D}$ to $2 \mathrm{D}$ projection within the lateral fissure), then spherically morphed to each subject. The labels were individually inspected (and filled if there were small holes in the label); then a boundary was delineated around each label on each subject's flattened auditory cortical patch.

ROI analyses. We quantified the similarity between frequency band response profiles driven by stimulus + attention (tonotopy) versus attention alone (attn-tono) in a "quilt" of small cortical surface-based ROIs that tiled the temporal plane. ROIs (see Fig. $5 B$ ) were created on a single subject's right and left hemisphere flattened patches by flooding all vertices within a $4 \mathrm{~mm}$ radius around a central selected vertex. Each of the ROIs (57 in the right hemisphere patch and 68 in the slightly larger left patch) were then spherically morphed to the other 7 subjects' flattened patches. Spurious ROI sampling on the edges of the patches was manually corrected on the original subject's inflated surface and remorphed to all other subjects. Each ROI was then projected into the registered nativespace EPI volume using Freesurfer's mri_label2vol (sampled from the gray-white boundary to 0.8 of the calculated cortical depth, with fillthresh set to 0.5 ). For each subject, within each ROI, we calculated the average parameter estimate for each frequency band for tonotopy, and combined stepped and randomized attn-tono conditions. For each ROI, we then ran a linear model with average tonotopy parameter estimates for the 5 frequency bands predicting average attn-tono parameter estimates for the same bands, including subjects as a random factor. The resulting partial $t$ statistic for each ROI was $z$-transformed and color-rendered in Figure $5 B$, with $p$ value thresholds Bonferroni-corrected to $p<0.05$ for the number of ROIs per hemisphere, and indicated by the white outline surrounding the set of ROIs that surpass this $z$-threshold. 


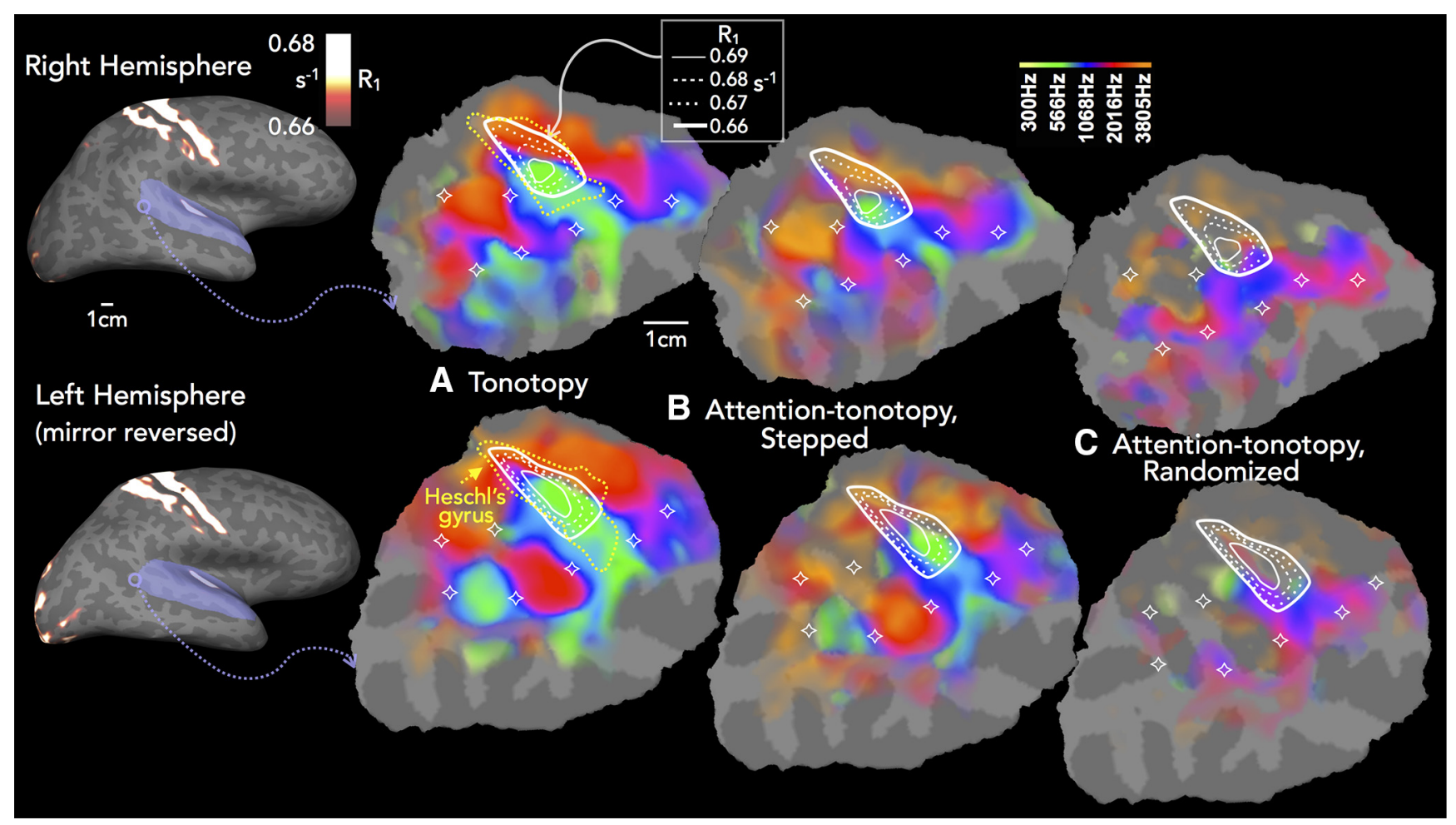

Figure 2. Group activation for tonotopy and attn-tono conditions, with $\mathrm{R}_{1}$ contours showing putative auditory core. Leftmost panel, Cortical surface-based group-averaged $\mathrm{R}_{1}$, projected on the lateral inflated surface of one subject. The left hemisphere is mirror-reversed to align cortical maps for visual comparison. For tonotopic map display, a patch of cortex, including the entire temporal plane (shown in purple on the inflated surface), was cut and flattened. $A-C$, Enlarged region, with isocontour lines showing quantitative $R_{1}$ values for the group-averaged putative auditory core and color maps showing group-averaged best frequency as a function of $(\boldsymbol{A})$ tonotopy, $(\boldsymbol{B})$ attn-tono (stepped), and $(\boldsymbol{C})$ attn-tono (randomized control) conditions. Stars represent fiduciary points to assist in visual comparisons of maps across conditions. Yellow dashed lines indicate the outline of Heschl's gyrus (in $\boldsymbol{A}$, from the individual subject whose cortical patch was used). Consistent with previous work, the tonotopic map is characterized by two pairs of three interlacing best-frequency fingers, with the high-frequency fingers (red/orange colormap) showing greatest frequency preference medially and extending laterally, where they meet interdigitated lower-frequency fingers (green/yellow colormap) extending lateral to medial, with the longest middle lower-frequency finger extending approximately halfway into auditory core. This pattern is evident in Fourier analysis-derived maps of the stepped attn-tono condition but not in the randomized control attn-tono condition, for which the attentional response was phase-cancelled. All maps are statistically masked by overall activation to tonotopy stimuli in each hemisphere (cluster-corrected $p<10^{-8}$, and gently shaded to show relative amplitude).

\section{Results}

\section{Fourier based analyses}

Stimulus-driven and attentionally driven tonotopic organization in human auditory core

As a necessary first step, we characterized basic tonotopic (stimulusdriven) organization in and immediately around myelin-estimated auditory core. The group-average $\mathrm{R}_{1}$-based estimates of myelination (inflated hemispheres, Fig. 2, leftmost panel) show that the highest $R_{1}$ values occur within primary somatomotor areas along the central sulcus, and in the typically keyhole-shaped presumptive "auditory core" lying along and immediately surrounding Heschl's gyrus. It is important to note that myelination varies within auditory core and that the lateral and medial borders are less sharply demarcated (for review, see Hackett et al., 2001; Dick et al., 2012). To show this variation, we plot isointensity $R_{1}$ contours in the cortical flat patches in Figure $2 A-C$ (with the curvaturebased boundaries of Heschl's gyrus overlaid in dotted lines). To help identify the discontinuities in $\mathrm{R}_{1}$ that would correspond to the putative borders of auditory core, we calculated the $\mathrm{R}_{1}$ gradient along the surface (Glasser et al., 2016). Lines drawn along the peak gradient amplitude (data not shown) corresponded well with the outermost $R_{1}$ isointensity contour in Figure $2\left(0.66 \mathrm{~s}^{-1}\right)$. It is important to note that the gradient at the lateral edge of presumptive core is quite shallow; and as in postmortem myelin stains, it is therefore more difficult to establish an unambiguous lateral border, as could be surmised from the greater lateral spread of the isointensity contours. The shape and size $(\sim 1.2 \mathrm{~cm} \times 2.4 \mathrm{~cm})$ of presumptive auditory core in this sample also agreed with the results from Dick et al. (2012) at the same $R_{1}$ threshold (with the latter average core slightly narrower; data not shown here).

The group-averaged topography of preferred frequency around auditory core has a typical arrangement (Dick et al., 2012; De Martino et al., 2015b), with the core surrounded by a high-frequency "V." Preferred frequency descends into the center of core (where $\mathrm{R}_{1}$ values are highest) before reversing and slowly ascending to mid-frequency preferred frequencies anterolaterally (and to some extent posterolaterally). Figure 3 shows tonotopic maps for each individual listener. In general, the relationship between auditory core and tonotopy group is conserved across listeners, but with some variability in the shape and extent of the isointensity $R_{1}$ contours. In particular, S2, S6 (right hemisphere), S7, and S8 (right hemisphere) had irregularly shaped and "blotchy" isointensity contours. Although there is a fair degree of individual variability in results from human postmortem cytoarchitectonic and myeloarchitectonic studies of auditory core and surrounding areas (Hackett et al., 2001; Sweet et al., 2005), this was somewhat greater than expected variation given other work in our laboratory (Dick et al., 2012; Lutti et al., 2014; Carey et al., 2017). As an independent estimate of primary auditory areas, we also morphed the Morosan et al. (2001) 3D probabilistic map of primary auditory areas (TE1.0) using previously established methods (see Materials and Methods); the outlines of the morphed labels corresponding to 


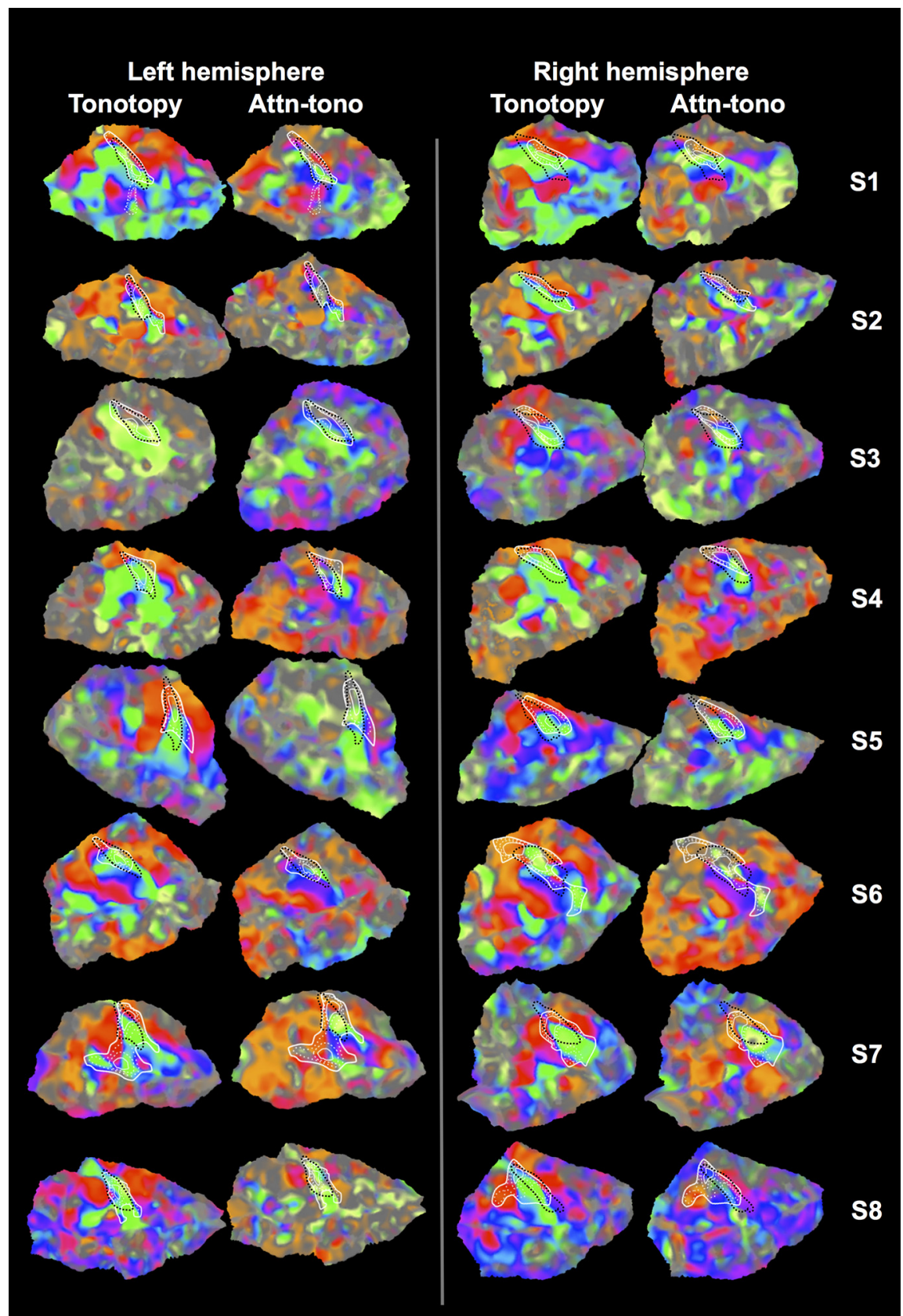

Figure 3. Individual subjects' tonotopy and attn-tono maps. Each subject's tonotopic and attn-tono (stepped) Fourier analysisderived maps are displayed on the same subject's flattened superior temporal cortical patch. White represents $R_{1}$ isocontours around presumptive auditory core. Thick solid lines indicate the lowest valued (outermost) $R_{1}$ isocontour. Thin solid lines indicate the highest (innermost) $R_{1}$ isocontour. Dashed lines indicate intermediate values. $R_{1}$ values differ somewhat across individuals. Dashed black lines indicate the outline of the cortical surface-morphed TE1.0 label, where the area inside the line contains vertices estimated to have a $p>0.3$ probability of falling within primary auditory cortex based on the Morosan et al. (2001) postmortem probability atlas (see Materials and Methods). Activation maps are gently shaded to show changes in response amplitude but are unthresholded for comparison with individual maps from previous studies (e.g., Da Costa et al., 2011).

$p>0.30$ of being within TE1.0 are overlaid in black dotted lines in Figure 3.

We then asked whether attn-tono mapping resembled the tonotopic case in and around auditory core. Here, the grouplevel spatial distribution of tonotopy is closely recapitulated when spectrally directed attention (stepped attn-tono condition) alone modulates activation (Fig. 2B). This holds true in and around the keyhole-shaped hyperintensity defining core, with a slight exception in the transition from higher to lower frequency preference in mid core. In contrast (and as expected), the group-level attn-tono response for the randomized control condition is much weaker (Fig. 2C), with almost no correspondence with the tonotopic map, despite being acoustically identical to stepped attn-tono but for the shuffling of the verbal prompt ordering that destroyed the consistent phase lag associated with specific frequency bands. The one potential exception is in and around posterolateral core, where there is a low-to-mid frequency progression that is similar in attn-tono and tonotopic maps, particularly in the left hemisphere. This may be due to the small ( $\sim 1$ octave) overall shift in spectral mean over the course of a stimulus cycle noted in Materials and Methods.

Stimulus-driven and attentionally driven tonotopic organization outside of auditory core

In line with results from previous fMRI studies (Talavage et al., 2004; Woods et al., 2009; Humphries et al., 2010; Barton et al., 2012; Dick et al., 2012; Moerel et al., 2012; Saenz and Langers, 2014; De Martino et al., 2015b; Thomas et al., 2015; Ahveninen et al., 2016; Leaver and Rauschecker, 2016; Riecke et al., 2017), there is stimulus-driven tonotopic mapping extending well beyond auditory core, spanning the temporal plane and continuing into the superior temporal sulcus. As shown in Figure $2 A$, the overall arrangement is characterized by two pairs of three interlacing best-frequency "fingers," with the high-frequency fingers (red/orange colormap) predominating medially and extending laterally, where they meet interdigitated lower-frequency fingers (green/yellow colormap) extending lateral to medial, with the longest "middle" lower-frequency finger extending approximately halfway into auditory core. Similar to tonotopy within auditory core, the overall pattern of group activation can be observed in the majority of individual subjects (Fig. 3), but there is also considerable individual variability in the complexity, topography, and extent of tonotopic and attn-tono mapping, similar to that observed in the fMRI studies cited above (as well as electrophysiological studies in a number of studies in macaque and owl monkey) (e.g., Merzenich and Brugge, 1973; Morel et al., 1993).

As can be seen in the maps in Figure $2 B$, the tonotopically aligned maps evoked by spectrally directed attention are also present in the majority of auditory cortex outside of auditory core. Again, the structure of the tonotopic map (as revealed by Fourier analysis) is abolished when the attentional cue is randomized, thereby eliminating any consistent relationship between attended frequency band and phase lag (Fig. 2C). 


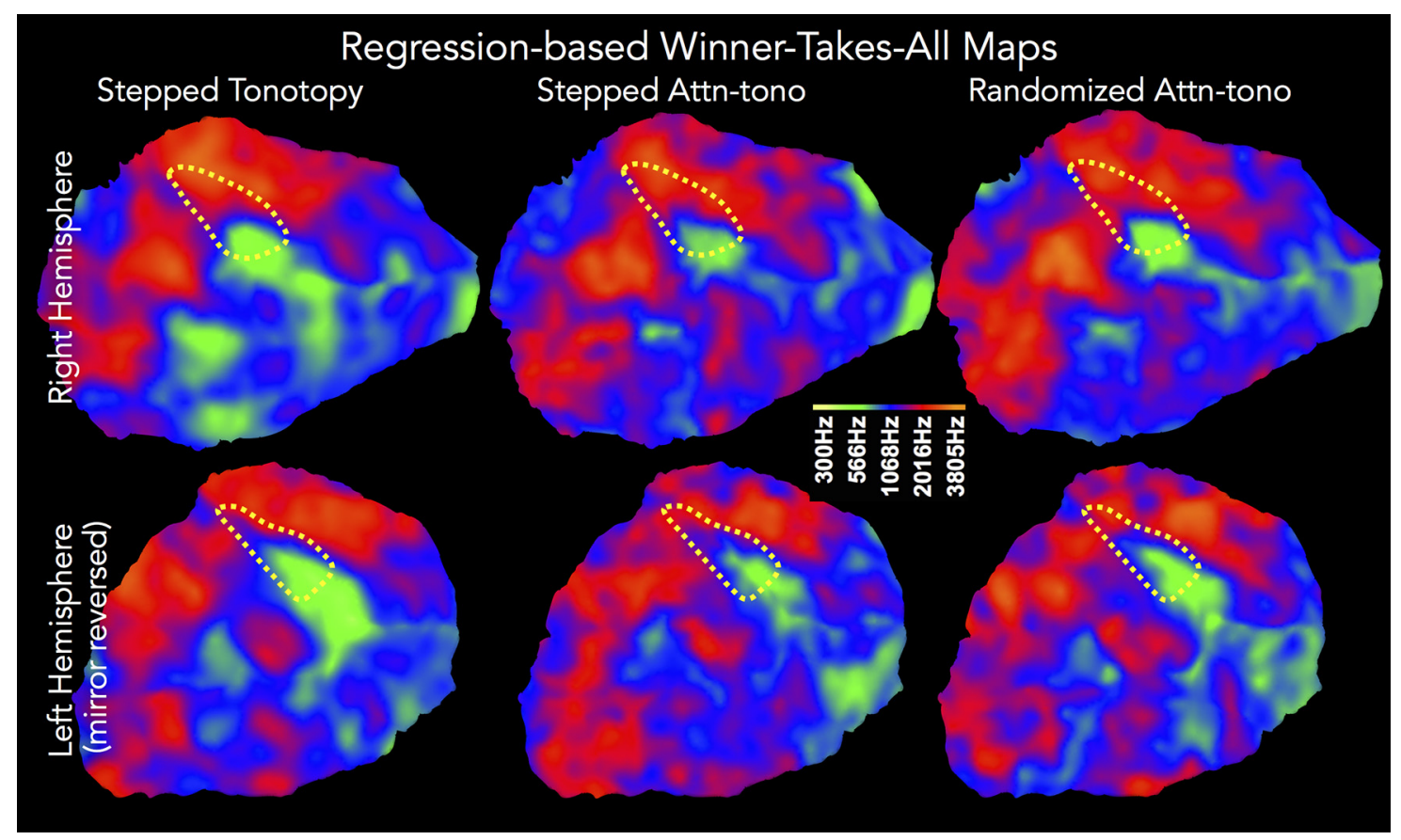

Figure 4. Comparison of responses in regression-based WTA maps, tonotopy, and attn-tono. Color maps projected onto the right (top panels) and left (bottom panels) hemisphere cortical patches (same as patches shown in Fig. 2, purple) show the cross-subject average best frequency band (WTA) for stepped tonotopic (left) stepped attn-tono (middle), and randomized attn-tono (right) conditions. With the regression-based approach, the randomized condition is also expected to evoke strong attentionally driven tonotopic maps. Dotted yellow line indicates the outermost $\mathrm{R}_{1}$ contour $\left(0.66 \mathrm{~s}^{-1}\right)$ around presumptive auditory core as shown in Figure 2 .

The similarity between the maps evoked by presentation of a single-frequency band (tonotopy) versus attention to one of two simultaneously presented frequency bands (stepped attn-tono) can also be seen in each individual subject (Fig. 3). As with the group-averaged data, there is a close correspondence in the progression of preferred frequencies across auditory cortex in individual subjects. The similarity between the tonotopic and attn-tono maps is particularly striking in Subjects 1, 2, 5, 6, and 7. The tonotopic organization of individual subjects demonstrated overall commonalities, but with notable differences, even between individual subjects' right and left hemispheres, particularly outside of auditory core (Humphries et al., 2010; Moerel et al., 2014; Saenz and Langers, 2014). However, individual peculiarities were replicated across tonotopic and attn-tono conditions. In some subjects, there was a surprising lack of strong tonotopic mapping (Subject 3 for which poor tonotopy may be due to greater EPI warping, and also Subject 4 for which low frequencies dominated the tonotopic maps). In summary, there was a strong correspondence between tonotopic and attn-tono maps at both the group and individual levels.

\section{Multiple regression analyses}

Winner-takes-all (WTA): maps of "stepped" versus "randomized" attention conditions, and quantitative concordance of tonotopic and attn-tono maps

In a complementary analysis, we used standard multiple regression techniques (see Materials and Methods) to estimate the BOLD response to each center frequency band when it was presented in isolation (tonotopy), versus when it was attended in the presence of a distractor band (attn-tono). This allowed us to make use of the attentionally driven signal in the randomized attn-tono condition and to combine these data with the results from the stepped attn-tono condition to increase statistical power. It also allowed us to verify that the attention effects generalize when listeners direct attention without the "crutch" of consistent stepping up or down across attended frequency bands.

The auditory cortical patches in Figure 4 show the crosssubject average WTA best frequency band (most positive-going BOLD response relative to resting baseline) maps for tonotopy and attn-tono conditions (with no shading for response amplitude). These are overlaid with the outermost $R_{1}$ isocontour (dashed yellow) corresponding to auditory core. As should be expected, the topography of the WTA maps essentially recapitulates the topography revealed by the phase-encoded analyses. The same holds true of the attn-tono WTA maps from both the stepped and, importantly, the randomized block conditions (Fig. 4); this result confirms that, even without the crutch of the stepping frequency band, listeners can direct their attention to specific frequency bands.

The WTA approach also allowed us to straightforwardly quantify the within-subject correspondence between voxelwise best frequency, as estimated by tonotopy and by attn-tono. Here, we coded each voxel in native space as a 1 when best frequency was identical in both conditions, and a 0 otherwise. We then resampled each subject's binary maps to their cortical surface, and then averaged across subjects to create a concordance map (Fig. 5A). These maps (statistically thresholded at vertexwise $p<$ 0.01 , with surface-cluster-corrected $\alpha$ of $p<0.001$ ) show that, across subjects, there was high concordance across best frequency maps evoked by stimulus and by attention across much of the temporal plane in both hemispheres, with little concordance in nonauditory areas. The extent of attentionally driven tonotopic mapping relative to overall tonotopicity is shown in the cortical patches below each concordance map in Figure $5 \mathrm{~A}$. Here, the outer contour of the significant ( $p<0.001$ clusterwise corrected) concordance map is overlaid on the phase-averaged group tono- 


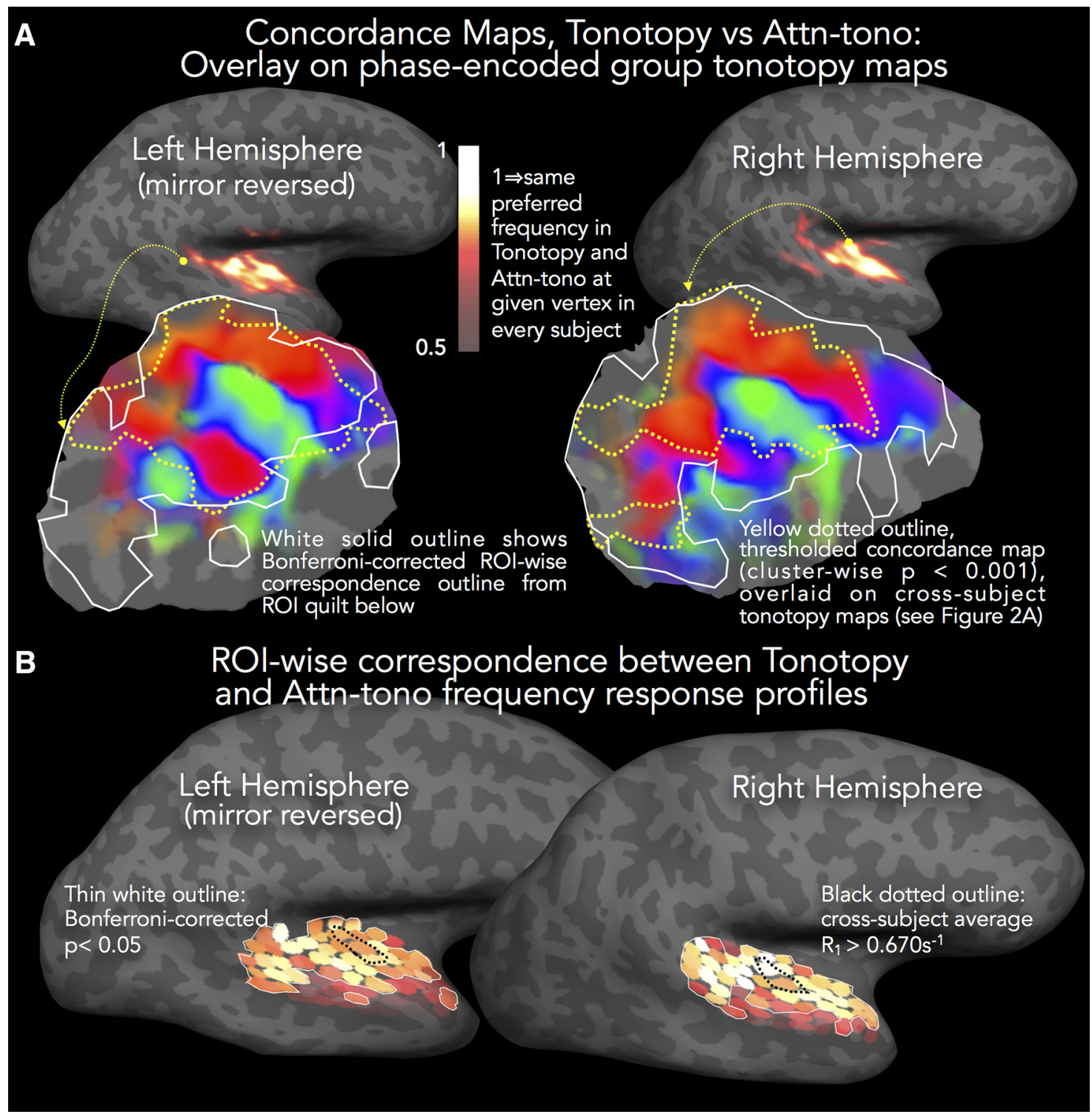

Figure 5. Comparison of tonotopy and attn-tono maps. A, Concordance maps are rendered in heatscale on the inflated hemispheres to illustrate the similarity in best frequency between tonotopic and attn-tono maps (the latter averaged over stepped and randomized blocks). These maps were calculated in two stages. First, in each subject's native EPI space, a voxel was coded as 1 if tonotopy and attn-tono stimuli evoked the same best frequency, and otherwise coded as 0 . Second, for each subject, the concordance maps were resampled to the individual's cortical surface and projected onto the unit icosahedron for cross-subject surface-based averaging, thereby creating a composite measure of agreement between tonotopy and attn-tono maps, weighted by the consistency of this agreement across subjects. The concordance maps are statistically masked with a cross-subject $t$ map, calculated versus chance agreement $(p=0.20)$ with a surface cluster correction of $p<0.001$ (vertexwise $p<0.01$, cluster threshold surface area $>74 \mathrm{~mm}^{2}$ ) (Hagler et al., 2006). To demonstrate the extent of tonotopically mapped cortex that is similarly mapped through spectrally directed attention, the phase-encoded tonotopy cortical patches from Fig. $2 A$ are overlaid with the outline of the thresholded concordance map shown by the yellow dotted line. White solid outline indicates the Bonferroni-corrected ROl-wise correspondence outline from the ROl quilt in B. B. Shading in each small ROI patch represents the zscore for the partial fit between tonotopy and attn-tono responses to each frequency band (with subjects as a random factor). Thin white outline indicates ROIs with significant $z$ scores (Bonferroni-corrected $p$ value threshold of $p<0.05$ ).

topy map (same as Fig. 2A). Averaging over all subjects, the majority of consistently tonotopically mapped cortex medial to the crown of the superior temporal gyrus (STG) shows preferredfrequency-aligned attn-tono maps, as does a small posterior cluster. In the left hemisphere, almost all consistently mapped tonotopic cortex also shows aligned attn-tono maps. However, it is important to note that there are considerable individual differences in regional best-frequency alignment across the tonotopy and attn-tono maps (as can be seen in Fig. 3).

Comparison of response profiles to all frequency bands across tonotopy and attn-tono

As has been shown previously (e.g., Moerel et al., 2013), hemodynamic responses to frequency in auditory cortex are not nec- essarily bandpass but can be more complex and multipeaked. Therefore, we also examined whether attention to a given frequency band in the presence of a distractor band recapitulates the more graded response to nonpreferred frequencies observed when that frequency band is presented in isolation. To do this, we created and surface-morphed a set of small cortical ROIs to each subject (Fig. 5B; see Materials and Methods) and quantified the similarity between the tonotopy and attn-tono response profiles in each ROI in each hemisphere by regressing the mean tonotopic parameter estimate for each frequency band against the attn-tono parameter estimate (with subjects as a random factor). We used this ROI quilt analysis (as opposed to a vertexwise one) to capture regional variation in cross-condition response profile similarity across 


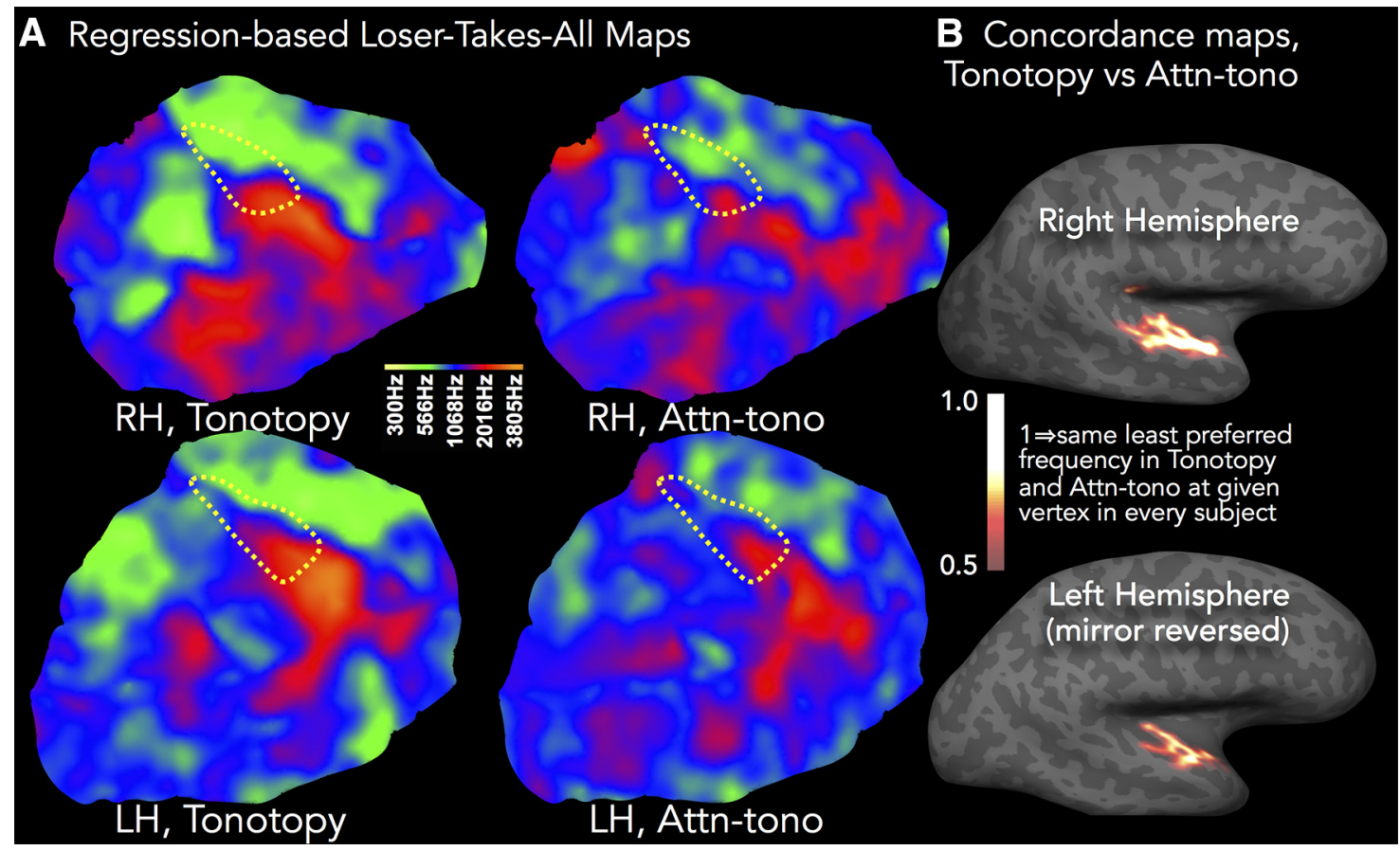

Figure 6. Comparison of responses in regression-based LTA maps, tonotopy, and attn-tono. $A$, The colormaps projected onto the same cortical patches as in Figures 2 and 4 show cross-subject group-average maps that depict the frequency band that drives the least activation compared with all other frequency bands (LTA) in tonotopy and attn-tono (stepped plus randomized blocks) conditions and in right and left hemispheres. As in Figure 4, the presumptive auditory core shown by the dashed yellow line depicting the outermost $R_{1}$ contour $\left(0.66 \mathrm{~s}^{-1}\right)$. B, The tonotopy versus attn-tono LTA concordance map was created as in Figure 5 A. The midpoint of the heatscale has been lowered slightly compared with Figure $5 A$, reflecting the overall somewhat lower concordance in the LTA maps compared with WTA. Dotted yellow $R_{1}$ isocontour is the same as in Figure 4.

subjects, which might be obscured by individual differences in tonotopic map topography and surface-based registration errors. This also reduced the number of statistical comparisons that must be corrected for, thus increasing power to detect effects.

The ROI analyses (Fig. 5) further support the results from the concordance maps from the WTA analyses (Fig. 4). The ROI analyses (Fig. $5 B$ ) show that individual subjects' tonotopy and attn-tono responses profiles are significantly associated across most of auditory cortex (all ROIs within the white border), with the exception of the most lateral aspects of the STG and upper bank of the superior temporal sulcus. Although there is a strong relationship between tonotopy and attn-tono response profiles of each subject within a given ROI, there is cross-subject variability in the particular shape of those response profiles, as suggested by the individual maps in Figure 3. There is a broad tendency for tonotopy/ attn-tono profile similarity to be strongest posteromedially in both hemispheres, and no clear indication that profile similarity is higher in auditory core (indeed, this is not the case in the left hemisphere). As shown by the white line on the tonotopic flat maps in Figure 5A, the area showing significant response profile similarity extends over the majority of cortex showing strong tonotopic mapping with topologic similarity across subjects. The response profile similarity extends into less tonotopically consistent regions medially and posteriorly but does not include the more posterolateral tonotopically mapped regions along the crown of the STG.

Loser-takes-all (LTA): maps of dis-preferred frequency Given the graded nature of frequency response preferences we observed, we suspected that there would be a large-scale topography associated with the minimum BOLD response across frequency, and that this topography would also be recapitulated by attention. Thus, we also performed a parallel LTA analysis, in which we coded voxels by the frequency band driving the minimum BOLD response (again relative to resting baseline) and analyzed as above. The average descriptive LTA maps show approximately opposite frequency responses compared with the WTA tonotopic maps, with higher-frequency band-preferring regions in the tonotopic map being least driven by lower-frequency bands, and vice versa (Fig. 6A). There is also some overlap in the mid-frequency-preferring regions, likely due to blurring of values when averaging subjects' integer-based maps. There is also quite close correspondence between the frequency band evoking the least response in the tonotopy (stimulus) condition and the smallest BOLD response evoked by attending to a given frequency band. The LTA concordance maps (Fig. $6 B$, statistical thresholding as in Fig. $5 \mathrm{~A}$ ) show that, in the right hemisphere, the alignment of tonotopic and attn-tono maps is greatest in more lateral and anterior auditory cortex, with qualitatively somewhat greater concordance more medially in the left hemisphere. The hemispheric difference and also the apparent qualitative contrast with the WTA concordance maps seen in Figures 4 and 6 are exaggerated by the clusterwise statistical thresholding combined with the overall slightly lower concordance in the LTA maps.

Difference in activation across auditory areas when best frequency is attended versus ignored

We also assessed the strength and consistency of BOLD-related frequency band-selective attention across subjects, and how the effect of attention varied with preferred or dis-preferred frequency. We first used a subject's native-space WTA map to establish each voxel's best frequency. Then, we assigned each voxel the parameter estimate for the difference in activation between attending to its best frequency in the presence of a distractor versus attending to the distractor and ignoring its best frequency. In other words, the value at each voxel was the estimated differ- 


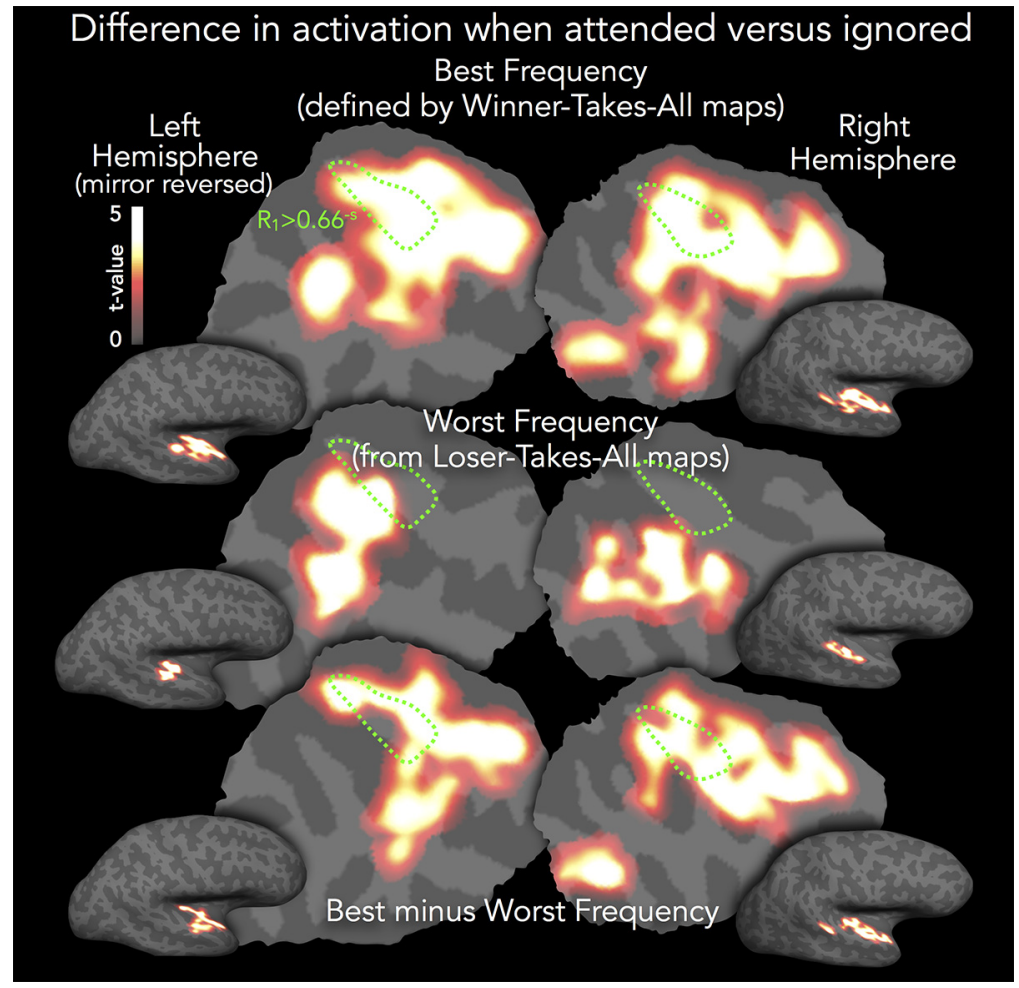

Figure 7. Comparison of maps when best frequency is attended versus ignored. The heatscale ( $t$ values, thresholded as in Fig. $5 A$ ) depicts the cross-subject cortical surface-based average difference in activation when the subject-specific best frequency band of each voxel was attended versus ignored. The dotted green $\mathrm{R}_{1}$ isocontour estimating auditory core is as in Figure 4 . versus preferred frequency band). This shows that the frequency-selective attentionrelated BOLD gain is strongly modulated by frequency preference and provides some evidence for models of multiplicative, and not additive, attentional gain (but see Discussion).

\section{Relationship of tonotopic and attn-tono map strength to MR-estimated myeloarchitecture}

Typically, assays of cortical myelination are used to differentiate the most highly myelinated cortical regions (e.g., auditory core, MT/V5, or V1) from adjacent regions. This is true whether cortical myelination is assessed using ex vivo "gold standard" approaches, such as Gallyas staining, or estimated through in vivo MRI T1-weighted/ T2-weighted ratio, quantitative $\mathrm{R}_{1}$, or magnetization transfer measures. However, more subtle myelination changes that occur throughout cortex may spatially correspond with changes in functional characteristics (Glasser et al., 2016; Wallace et al., 2016). For instance, recent combined fMRI and high-resolution quantitative MR show that slight reductions in cortical myelination in primary somatomotor cortex reliably occur at the border between face and hand areas (Kuehn et al., 2017).

ence in activation between attending to, versus ignoring, its best frequency in the presence of other frequency bands. We repeated this process to estimate the parallel attention effect for each voxel's "worst" frequency (using the corresponding LTA map). We then resampled each subject's native-space attention maps to her/his cortical surface to allow for surface-based cross-subject averaging and statistical testing (all again with a vertexwise $p<$ 0.01 threshold and surface-cluster-corrected $\alpha$ of $p<0.001$ ). Figure 7 (top row) shows that, across subjects, there was significantly greater activation across most of auditory cortex when best frequency was attended versus ignored. The widespread attention effect included all of $\mathrm{R}_{1}$-estimated auditory core (outlined in green), extending from the inferior circular sulcus laterally to the upper bank of the superior temporal sulcus, and anteroposteriorly from the temporal pole onto the planum temporale. By contrast, there were relatively few regions where attention to a voxel's least-preferred frequency band evoked greater activation than when the same frequency band was the distractor. Attending to a voxel's least-preferred frequency band only significantly increased activation along the posterior lateral STG in both hemispheres, extending more medially in the left and more anteriorly in the right (Fig. 7, middle row). A direct comparison between these maps (cross-subject $t$ test on the difference of differences, Fig. 7, bottom row) showed that there were considerable regional differences in activation between attending to a voxel's preferred versus dis-preferred frequency band. In both hemispheres, there was greater activation across most of the anterior temporal plane when attention was directed to the preferred versus dis-preferred frequency; in the right hemisphere, this effect extended throughout the temporal plane, as well as including a patch in the posterior STG. There were no regions in which the converse effect was observed (greater attend $>$ distract activation for dis-preferred
Here, we asked whether the change in the degree to which cortex showed a strong frequency band preference (i.e., the amplitude of the phase-encoded tonotopic or attn-tono signal) spatially corresponded with changes in myelination as assessed by quantitative $\mathrm{R}_{1}$ (within a 4-mm-radius disk that roved across the entire cortical surface). The cross-subject-average normalized covariance map in Figure $8 \mathrm{~A}$ shows that there is a shared local gradient in tonotopic amplitude and $\mathrm{R}_{1}$ along the entire inferior circular sulcus and the anterior part of the STG, where tonotopic amplitude and $\mathrm{R}_{1}$ drop in tandem over a narrow band of cortex. There is also negative local spatial covariance between tonotopic amplitude and $\mathrm{R}_{1}$ within the center of auditory cortex, where tonotopic amplitude increases but $\mathrm{R}_{1}$ remains relatively stable. There is also some tonotopic/ $R_{1}$ spatial covariance within and around the central sulcus; these regions showed considerably less overall amplitude in tonotopic response, but one that spatially covaries with changes in $\mathrm{R}_{1}$.

To test the replicability of this novel tonotopy-versus- $\mathrm{R}_{1}$ searchlight cross-correlation, we reanalyzed $\mathrm{R}_{1}$ and tonotopy data from a previous study (Dick et al., 2012) that used a different tonotopic stimulus (bandpass-filter-swept nonlinguistic vocalizations) and a slightly different multiparameter mapping protocol. Despite these methodological differences, we found a very similar pattern of tonotopic/ $\mathrm{R}_{1}$ positive local spatial covariance within the circular sulcus and along the lateral STG, with negative spatial covariance again in the center of auditory cortex (Fig. $8 B$ ). The shared and relatively steep anterolateral and medial gradients in putative myelination and degree of frequency specificity, observed in two independently acquired datasets, suggest a shared functional and myeloarchitectonic border, possibly similar in character to those reported recently relating resting state, standard task activation, 
and T1-weighted/T2-weighted derived myelination estimates across cortex (Glasser et al., 2016; Kuehn et al., 2017).

As seen in Figure $8 C$, the spatial relationship between local $\mathrm{R}_{1}$ and attn-tono amplitude changes is much less clear. Here, there is a weak relationship within and around auditory cortex that is only observed within the circular sulcus (particularly in the right hemisphere). There are also stripes of spatial covariation along the banks of the central sulcus, although not closely aligned with the pattern observed with the tonotopy versus $R_{1}$ covariance maps. Although very preliminary, these results suggest that changes in the degree of spectral attentional modulation in auditory cortex are not strongly linked to the underlying myeloarchitecture, and stand in contrast to the consistent spatial association in lateral and medial auditory cortex between local changes in $\mathrm{R}_{1}$ and the strength of stimulus-driven frequency response preference.

In summary, everyday listening ordinarily takes place in rich soundscapes within multiple, simultaneous sound sources contributing to the overlapping mix of sound waves that arrives at the ears. Auditory attention is crucial to sorting out the mix. Listeners direct attentional focus to a sound source, or even to specific acoustic dimensions within a single sound source, to zero in on auditory information that is diagnostic in guiding behavior.

We asked how endogenous attention directed to specific acoustic frequency bands modulates human auditory cortical activity. Using high-resolution quantitative MRI and a novel fMRI paradigm for driving sustained selective attention within specific frequency bands, we established effects of spectrally specific attention in myeloarchitectonically estimated human auditory core. These effects extend across the majority of tonotopically mapped auditory cortex and are apparent in individual listeners. Sensorydriven best-frequency tonotopic maps align with attentionally driven maps across much of the temporal plane, with poor concordance in nonauditory areas. Individual tonotopic and attntono maps show correlated idiosyncracies. The frequency bands that evoke the least BOLD response from input and from attention also exhibit close spatial correspondence. There is greater activation across most of auditory cortex when best frequency is attended, versus ignored. Finally, there is local spatial correspondence in multiple auditory regions between the degree of $\mathrm{R}_{1}$-estimated myelination and the strength of the frequency band-selective fMRI response for tonotopic stimuli.

\section{Discussion}

Human auditory core exhibits attentionally driven tonotopic organization

Previous findings showed similar stimulus-driven and attentionally driven frequency preference in and around Heschl's gyrus, a macroanatomical landmark associated with primary auditory areas (Da Costa et al., 2013; Riecke et al., 2017). Here, we demonstrate that, within quantitative- $\mathrm{R}_{1}$-defined primary auditory areas, the attentionally driven maps in each hemisphere are very similar to the detailed tonotopic maps in the same subjects. As shown by comparison maps across the acoustically identical stepped and randomized attn-tono conditions (Figs. 2, 4), the alignment between tonotopic and attention maps depends on allocation of attention to the cued frequency band, not perceptual interference or other stimulus-driven effects. The fact that there is considerable, high-level attentional modulation within primary auditory areas is interesting given previous results suggesting more limited attentional topographic modulation in primary auditory (Atiani et al., 2014) and visual (Saygin and Sereno, 2008) cortex, compared with more robust attentional modulation in areas immediately adjacent to primary ones.

\section{Attentionally driven tonotopic organization extends across much of auditory cortex}

We also find strong evidence for tonotopically mapped spectrally directed attention in much of auditory cortex, particularly along the lateral STG (potentially analogous to lateral auditory belt and parabelt regions in macaque) (Hackett, 2007). In addition to the concordance in and around auditory core, the most consistent group-level alignment of these maps lies lateral to auditory core, with each map characterized by three higher-to-lower bestfrequency band traversals, moving from posterior to anterior approximately along the STG.

This pattern suggests a cross-species parallel to results reported in ferret (Atiani et al., 2014), where task-evoked attentional modulation of frequency-tuned neurons is particularly 
strong in nonprimary (dPEG) tonotopically mapped auditory areas. In this regard, the stimulus complexity, variability, and memory demands of the current task may have helped to drive attentional response in these more lateral and anterior areas. Our results are consistent with a human fMRI comparison of crossmodal attentional effects (Petkov et al., 2004), which showed greater activation in lateral auditory regions when attention was directed to a demanding auditory repetition detection task than when the same sounds were played as subjects performed a demanding visual detection task. However, our results differ from these studies to some degree in that attentionally driven tonotopic modulation in auditory core was also robust (similar to cross-modal attention studies in macaque A1) (O'Connell et al., 2014) and primary auditory areas (De Martino et al., 2015a), and did not differ significantly from that in lateral belt.

There was good correspondence between the voxelwise best frequency band for tonotopy and attn-tono in individual listeners. Like several prior studies (Humphries et al., 2010; Moerel et al., 2014; Saenz and Langers, 2014; Brewer and Barton, 2016; Leaver and Rauschecker, 2016), we observed quite substantial variation in the detailed topography of tonotopy across individuals (but compare Ahveninen et al., 2016). It is especially noteworthy that attn-tono recapitulated these topographic idiosyncrasies (as observed in the concordance analyses, Figs. $4 B, 5 B)$.

It is intriguing that there was a systematic frequency bandassociated topography not only of best frequency but also of dis-preferred frequency and, also, that the frequency-selective attenuation of BOLD gain (relative to other frequencies) can be recapitulated by selective attention to that frequency band in the presence of other spectral information. One could speculate that this map structure might be a population-level reflection of an "inhibitory surround" structure observed in some electrophysiology studies (Calford and Semple, 1995; Sutter et al., 1999; but compare Wehr and Zador, 2003), with the frequency band driving the least BOLD response corresponding to the deepest trough in an asymmetric surround, an effect that could drive the very similar tonotopic and attn-tono graded frequency response preferences revealed in the multiple ROI analysis (Fig. 5B).

Here, the average frequency response profile evoked by the single-band tonotopic stimuli was recapitulated by attention to the same frequency bands in the context of distractors. Prior human neuroimaging research has been consistent with the possibility that the shape of the frequency response in and around Heschl's gyrus is attentionally modulated in a bandpass manner that relies on amplification rather than attenuation (Riecke et al., 2017). Based on results from a larger number of spectral bands, the current findings suggest that, at least at a more macroscopic scale, spectrally directed attention modulates cortical activity in a more graded fashion, with the shape of the attentional response to both preferred and less-preferred frequency bands similar to that evoked by stimulus alone, a contention supported by the alignment of the LTA tonotopic and attn-tono maps (Fig. 6). That is, the frequency band that drives the smallest fMRI response when presented alone is also the frequency band that elicits the least activation when attended in the presence of a distractor. A better understanding of the mechanisms underlying these maps will require more fine-grained characterization of frequency-directed attentional modulation, preferably at very high spectral and temporal resolution (Moerel et al., 2013, 2014; Lutti et al., 2014; Ahveninen et al., 2016) that might also help to unveil corticaldepth-specific attentional effects (De Martino et al., 2015a). In particular, it will be important to see whether different fMRI tasks, using more complex naturalistic sounds, or more or less abstract cues to frequency, can mimic the task-, valence-, and context-dependent effects observed in nonhuman animal cortical auditory receptive fields, where the character of the "contrastenhancing" modulations differs markedly with experimental manipulation (Fritz et al., 2005, 2007a, b; David et al., 2012; Atiani et al., 2014; Kuchibhotla et al., 2017). It is worth noting that task-related modulation of frequency-selective attentional effects has long been of interest in human auditory psychophysics (Greenberg and Larkin, 1968; Scharf et al., 1987; Scharf, 1989; Moore et al., 1996; Green and McKeown, 2001).

\section{There is correspondence between local change in $R_{1}$-estimated myelination and the strength of fMRI-assessed relative frequency selectivity}

We found that the change in the degree to which a small $(4 \mathrm{~mm}$ radius) patch of cortex shows strong frequency preferences in tonotopy was positively spatially correlated with its degree of myelination as estimated by $R_{1}$. The strength of the correlation was anatomically specific, marking the medial border of auditory cortex (within the circular sulcus) and revealing a potential anatomical index of "processing style" (from more to less tonotopically mapped) along anterolateral STG. We found this pattern to hold true in the data from the current study as well as in an independent cohort scanned with quite different tonotopic stimuli and with multiparameter maps acquired on a different scanner model, with different sequence settings (Fig. 8C). Although there was a relatively reliable pattern of $\mathrm{R}_{1}$-tonotopy correspondence at a group level, there was some notable individual variation in local shared $R_{1}$ /tonotopy gradients relative to gyral anatomy. Thus, these patterns may be more useful than curvature for establishing areal borders on an individual subject basis, particularly when there is no obvious sharp change in a single measure (for discussion, see also Glasser et al., 2016). Such work holds promise for generating novel hypotheses for more intensively characterized species (e.g., mouse, ferret, or marmoset), particularly in tandem with imaging techniques that that can cover multiple cortical areas simultaneously.

\section{Future directions}

In the current study, we limited our investigation to broadly defined auditory cortex, where there was good evidence for systematic tonotopic representation from a number of previous studies (Talavage and Edmister, 2004; Hackett, 2007; Moerel et al., 2013, 2014; Saenz and Langers, 2014; Leaver and Rauschecker, 2016). In future research, it will be informative to examine interactions with several frontal regions whose potential analogs are known to have direct feedforward and feedback connections in macaque monkeys (Romanski and Goldman-Rakic, 2002), and where in ferret there are clear modulatory influences on primary and nonprimary auditory cortex during learning (Atiani et al., 2014; Shamma and Fritz, 2014). Similar to recent work in vision (Klein et al., 2014; Puckett and DeYoe, 2015), it will also be useful to establish the shape of the attentional population receptive field, and how this varies across auditory areas and relates to stimulusdriven auditory population receptive field size (Thomas et al., 2015). Finally, following on from our own pilot work, it will be exciting to explore whether higher-level auditory regionalization may follow along some of the "fault lines" revealed by shared local tonotopic and myelin gradients, and whether or not more sophisticated and fine-grained spectral attentional manipulations may reveal a relationship between the degree of attentional malleability and underlying cortical architecture and circuitry. 


\section{References}

Ahveninen J, Chang WT, Huang S, Keil B, Kopco N, Rossi S, Bonmassar G, Witzel T, Polimeni JR (2016) Intracortical depth analyses of frequencysensitive regions of human auditory cortex using 7TfMRI. Neuroimage 143:116-127. CrossRef Medline

Atiani S, David SV, Elgueda D, Locastro M, Radtke-Schuller S, Shamma SA, Fritz JB (2014) Emergent selectivity for task-relevant stimuli in higherorder auditory cortex. Neuron 82:486-499. CrossRef Medline

Barascud N, Pearce MT, Griffiths TD, Friston KJ, Chait M (2016) Brain responses in humans reveal ideal observer-like sensitivity to complex acoustic patterns. Proc Natl Acad Sci U S A 113:E616-E625. CrossRef Medline

Barton B, Venezia JH, Saberi K, Hickok G, Brewer AA (2012) Orthogonal acoustic dimensions define auditory field maps in human cortex. Proc Natl Acad Sci U S A 109:20738-20743. CrossRef Medline

Brewer AA, Barton B (2016) Maps of the auditory cortex. Annu Rev Neurosci 39:385-407. CrossRef Medline

Calford MB, Semple MN (1995) Monaural inhibition in cat auditory cortex. J Neurophysiol 73:1876-1891. Medline

Callaghan MF, Helms G, Lutti A, Mohammadi S, Weiskopf N (2015) A general linear relaxometry model of R1 using imaging data. Magn Reson Med 73:1309-1314. CrossRef Medline

Carey D, Caprini F, Allen M, Lutti A, Weiskopf N, Rees G, Callaghan MF, Dick F (2017) Quantitative MRI provides markers of intra-, inter-regional, and age-related differences in young adult cortical microstructure. bioRxiv 139568.

Cox RW (2012) AFNI: what a long strange trip it's been. Neuroimage 62: 743-747. CrossRef Medline

Da Costa S, van der Zwaag W, Marques JP, Frackowiak RS, Clarke S, Saenz M (2011) Human primary auditory cortex follows the shape of Heschl's gyrus. J Neurosci 31:14067-14075. CrossRef Medline

Da Costa S, van Der Zwaag W, Miller LM, Clarke S, Saenz M (2013) Tuning in to sound: frequency-selective attentional filter in human primary auditory cortex. J Neurosci 33:1858-1863. CrossRef Medline

Dale AM, Sereno MI (1993) Improved localization of cortical activity by combining EEG and MEG with MRI cortical surface reconstruction: a linear approach. J Cogn Neurosci 5:162-176. CrossRef Medline

David SV, Fritz JB, Shamma SA (2012) Task reward structure shapes rapid receptive field plasticity in auditory cortex. Proc Natl Acad Sci U S A 109:2144-2149. CrossRef Medline

De Martino F, Moerel M, Ugurbil K, Goebel R, Yacoub E, Formisano E (2015a) Frequency preference and attention effects across cortical depths in the human primary auditory cortex. Proc Natl Acad Sci U S A 112: 16036-16041. CrossRef Medline

De Martino F, Moerel M, Xu J, van de Moortele PF, Ugurbil K, Goebel R, Yacoub E, Formisano E (2015b) High-resolution mapping of myeloarchitecture in vivo: localization of auditory areas in the human brain. Cerebral Cortex 25:3394-3405. CrossRef Medline

Dick F, Tierney AT, Lutti A, Josephs O, Sereno MI, Weiskopf N (2012) In vivo functional and myelo-architectonic mapping of human primary auditory areas. J Neurosci 32:16095-16105. CrossRef Medline

Dinse J, Härtwich N, Waehnert MD, Tardif CL, Schäfer A, Geyer S, Preim B, Turner R, Bazin PL (2015) A cytoarchitecture-driven myelin model reveals area-specific signatures in human primary and secondary areas using ultra-high resolution in-vivo brain MRI. Neuroimage 114:71-87. CrossRef Medline

Engel SA (2012) The development and use of phase-encoded functional MRI designs. Neuroimage 62:1195-1200. CrossRef Medline

Engel SA, Rumelhart DE, Wandell BA, Lee AT, Glover GH, Chichilnisky EJ, Shadlen MN (1994) fMRI of human visual cortex. Nature 369:525. CrossRef Medline

Feinberg DA, Setsompop K (2013) Ultra-fast MRI of the human brain with simultaneous multi-slice imaging. J Magn Reson 229:90-100. CrossRef Medline

Feinberg DA, Moeller S, Smith SM, Auerbach E, Ramanna S, Gunther M,Glasser MF, Miller KL, Ugurbil K, Yacoub E (2010) Multiplexed echo planar imaging for sub-second whole brain fMRI and fast diffusion imaging. PLoS One 5:e15710. CrossRef Medline

Fischl B, Sereno MI, Tootell RB, Dale AM (1999) High-resolution intersubject averaging and a coordinate system for the cortical surface. Hum Brain Mapp 8:272-284. CrossRef Medline

Fritz JB, Elhilali M, Shamma SA (2005) Differential dynamic plasticity of A1 receptive fields during multiple spectral tasks. J Neurosci 25:7623-7635. CrossRef Medline

Fritz JB, Elhilali M, David SV, Shamma SA (2007a) Does attention play a role in dynamic receptive field adaptation to changing acoustic salience in A1? Hear Res 229:186-203. CrossRef Medline

Fritz JB, Elhilali M, Shamma SA (2007b) Adaptive changes in cortical receptive fields induced by attention to complex sounds. J Neurophysiol 98: 2337-2346. CrossRef Medline

Fritz JB, David SV, Radtke-Schuller S, Yin P, Shamma SA (2010) Adaptive, behaviorally gated, persistent encoding of task-relevant auditory information in ferret frontal cortex. Nat Neurosci 13:1011-1019. CrossRef Medline

Glasser MF, Coalson TS, Robinson EC, Hacker CD, Harwell J, Yacoub E, Ugurbil K, Andersson J, Beckmann CF, Jenkinson M, Smith SM, Van Essen DC (2016) A multi-modal parcellation of human cerebral cortex. Nature 536:171-178. CrossRef Medline

Green TJ, McKeown JD (2001) Capture of attention in selective frequency listening. J Exp Psychol Hum Percept Perform 27:1197-1210. CrossRef Medline

Greenberg GZ, Larkin WD (1968) Frequency-response characteristic of auditory observers detecting signals of a single frequency in noise: the probesignal method. J Acoust Soc Am 44:1513-1523. CrossRef Medline

Greve DN, Fischl B (2009) Accurate and robust brain image alignment using boundary-based registration. Neuroimage 48:63-72. CrossRef Medline

Hackett TA (2007) Organization and correspondence of the auditory cortex of humans and nonhuman primates. Evolution of the nervous system (Kaas JH, ed). Academic Press: San Diego, CA, pp 109-119.

Hackett TA, Preuss TM, Kaas JH (2001) Architectonic identification of the core region in auditory cortex of macaques, chimpanzees, and humans. J Comp Neurol 441:197-222. CrossRef Medline

Hagler DJ Jr, Sereno MI (2006) Spatial maps in frontal and prefrontal cortex. Neuroimage 29:567-577. CrossRef Medline

Hagler D Jr, Saygin AP, Sereno MI (2006) Smoothing and cluster thresholding for cortical surface-based group analysis of fMRI data. Neuroimage 33:1093-1103. CrossRef Medline

Hagler DJ Jr, Riecke L, Sereno MI (2007) Parietal and superior frontal visuospatial maps activated by pointing and saccades. Neuroimage 35:15621577. CrossRef Medline

Helms G, Dathe H, Dechent P (2008) Quantitative FLASH MRI at 3T using a rational approximation of the Ernst equation. Magn Reson Med 59:667672. CrossRef Medline

Helms G, Draganski B, Frackowiak R, Ashburner J, Weiskopf N (2009) Improved segmentation of deep brain grey matter structures using magnetization transfer (MT) parameter maps. Neuroimage 47:194-198. CrossRef Medline

Herdener M, Esposito F, Scheffler K, Schneider P, Logothetis NK, Uludag K, Kayser C (2013) Spatial representations of temporal and spectral sound cues in human auditory cortex. Cortex 49:2822-2833. CrossRef Medline

Herrmann B, Henry MJ, Obleser J (2013) Frequency-specific adaptation in human auditory cortex depends on the spectral variance in the acoustic stimulation. J Neurophysiol 109:2086-2096. CrossRef Medline

Humphries C, Liebenthal E, Binder JR (2010) Tonotopic organization of human auditory cortex. Neuroimage 50:1202-1211. CrossRef Medline

Idemaru K, Holt LL (2011) Word recognition reflects dimension-based statistical learning. J Exp Psychol Hum Percept Perform 37:1939-1956. CrossRef Medline

Kastner S, Ungerleider LG (2000) Mechanisms of visual attention in the human cortex. Annu Rev Neurosci 23:315-341. CrossRef Medline

Klein BP, Harvey BM, Dumoulin SO (2014) Attraction of position preference by spatial attention throughout human visual cortex. Neuron 84: 227-237. CrossRef Medline

Kuchibhotla KV, Gill JV, Lindsay GW, Papadoyannis ES, Field RE, Sten TA, Miller KD, Froemke RC (2017) Parallel processing by cortical inhibition enables context-dependent behavior. Nat Neurosci 20:62-71. CrossRef Medline

Kuehn E, Dinse J, Jakobsen E, Long X, Schäfer A, Bazin PL, Villringer A, Sereno MI, Margulies DS (2017) Body topography parcellates human sensory and motor cortex. Cereb Cortex 27:3790-3805. CrossRef Medline

Langers DR, van Dijk P (2012) Mapping the tonotopic organization in human auditory cortex with minimally salient acoustic stimulation. Cereb Cortex 22:2024-2038. CrossRef Medline

Langers DR, Krumbholz K, Bowtell RW, Hall DA (2014) Neuroimaging 
paradigms for tonotopic mapping: I. The influence of sound stimulus type. Neuroimage 100:650-662. CrossRef Medline

Leaver AM, Rauschecker JP (2016) Functional topography of human auditory cortex. J Neurosci 36:1416-1428. CrossRef Medline

Lutti A, Hutton C, Finsterbusch J, Helms G, Weiskopf N (2010) Optimization and validation of methods for mapping of the radiofrequency transmit field at 3T. Magn Reson Med 64:229-238. CrossRef Medline

Lutti A, Stadler J, Josephs O, Windischberger C, Speck O, Bernarding J, Hutton C, Weiskopf N (2012) Robust and fast whole brain mapping of the RF transmit field B1 at 7T. PLoS One 7:e32379. CrossRef Medline

Lutti A, Dick F, Sereno MI, Weiskopf N (2014) Using high-resolution quantitative mapping of R1 as an index of cortical myelination. Neuroimage 93:176-188. CrossRef Medline

Merzenich MM, Brugge JF (1973) Representation of the cochlear partition of the superior temporal plane of the macaque monkey. Brain Res 50:275296. CrossRef Medline

Moerel M, De Martino F, Formisano E (2012) Processing of natural sounds in human auditory cortex: tonotopy, spectral tuning, and relation to voice sensitivity. J Neurosci 32:14205-14216. CrossRef Medline

Moerel M, De Martino F, Santoro R, Ugurbil K, Goebel R, Yacoub E, Formisano E (2013) Processing of natural sounds: characterization of multipeak spectral tuning in human auditory cortex. J Neurosci 33:1188811898. CrossRef Medline

Moerel M, De Martino F, Formisano E (2014) An anatomical and functional topography of human auditory cortical areas. Front Neurosci 8:225. CrossRef Medline

Moore BC, Hafter ER, Glasberg BR (1996) The probe-signal method and auditory-filter shape: results from normal- and hearing-impaired subjects. J Acoust Soc Am 99:542-552. CrossRef Medline

Morel A, Garraghty PE, Kaas JH (1993) Tonotopic organization, architectonic fields, and connections of auditory cortex in macaque monkeys. J Comp Neurol 335:437-459. CrossRef Medline

Morosan P, Rademacher J, Schleicher A, Amunts K, Schormann T, Zilles K (2001) Human primary auditory cortex: cytoarchitectonic subdivisions and mapping into a spatial reference system. Neuroimage 13:684-701. CrossRef Medline

O’Connell MN, Barczak A, Schroeder CE, Lakatos P (2014) Layer specific sharpening of frequency tuning by selective attention in primary auditory cortex. J Neurosci 34:16496-16508. CrossRef Medline

Oh J, Kwon JH, Yang PS, Jeong J (2013) Auditory imagery modulates frequency-specific areas in the human auditory cortex. J Cogn Neurosci 25:175-187. CrossRef Medline

Paltoglou AE, Sumner CJ, Hall DA (2009) Examining the role of frequency specificity in the enhancement and suppression of human cortical activity by auditory selective attention. Hear Res 257:106-118. CrossRef Medline

Petkov CI, Kang X, Alho K, Bertrand O, Yund EW, Woods DL (2004) Attentional modulation of human auditory cortex. Nat Neurosci 7:658663. CrossRef Medline

Pierpaoli C (2010) Quantitative brain MRI. Top Magn Reson Imaging 21: 63. CrossRef Medline

Preibisch C, Deichmann R (2009) Influence of RF spoiling on the stability and accuracy of T1 mapping based on spoiled FLASH with varying flip angles. Magn Reson Med 61:125-135. CrossRef Medline

Puckett AM, DeYoe EA (2015) The attentional field revealed by single-voxel modeling of fMRI time courses. J Neurosci 35:5030-5042. CrossRef Medline

Rao AA, Talavage TM (2005) Reliability of phase-encode mapping in the presence of spatial non-stationarity of response latency. Neuroimage 28: 563-578. CrossRef Medline

Riecke L, Peters JC, Valente G, Kemper VG, Formisano E, Sorger B (2017) Frequency-selective attention in auditory scenes recruits frequency representations throughout human superior temporal cortex. Cereb Cortex 27:3002-3014. CrossRef Medline

Romanski LM, Goldman-Rakic PS (2002) An auditory domain in primate prefrontal cortex. Nat Neurosci 5:15-16. CrossRef Medline

Saenz M, Langers DR (2014) Tonotopic mapping of human auditory cortex. Hear Res 307:42-52. CrossRef Medline

Saygin AP, Sereno MI (2008) Retinotopy and attention in human occipital, temporal, parietal, and frontal cortex. Cereb Cortex 18:2158-2168. CrossRef Medline

Scharf B (1989) Spectral specificity in auditory detection: The effect of listening on hearing. J Acoust Soc Jpn 10:309-317.

Scharf B, Quigley S, Aoki C, Peachey N, Reeves A (1987) Focused auditory attention and frequency selectivity. Percept Psychophys 42:215-223. CrossRef Medline

Schönwiesner M, Zatorre RJ (2009) Spectro-temporal modulation transfer function of single voxels in the human auditory cortex measured with high-resolution fMRI. Proc Natl Acad Sci U S A 106:14611-14616. CrossRef Medline

Schwarzkopf DS, Song C, Rees G (2011) The surface area of human V1 predicts the subjective experience of object size. Nat Neurosci 14:28-30. CrossRef Medline

Sereno MI, Huang RS (2006) A human parietal face area contains aligned head-centered visual and tactile maps. Nat Neurosci 9:1337-1343. CrossRef Medline

Sereno MI, Dale AM, Reppas JB, Kwong KK, Belliveau JW, Brady TJ, Rosen BR, Tootell RB (1995) Borders of multiple visual areas in humans revealed by functional magnetic resonance imaging. Science 268:889-893 . CrossRef Medline

Sereno MI, Lutti A, Weiskopf N, Dick F (2013) Mapping the human cortical surface by combining quantitative $\mathrm{T}(1)$ with retinotopy. Cereb Cortex 23:2261-2268. CrossRef Medline

Shamma S, Fritz J (2014) Adaptive auditory computations. Curr Opin Neurobiol 25:164-168. CrossRef Medline

Smith SM, Jenkinson M, Woolrich MW, Beckmann CF, Behrens TE, Johansen-Berg H, Bannister PR, De Luca M, Drobnjak I, Flitney DE, Niazy RK, Saunders J, Vickers J, Zhang Y, De Stefano N, Brady JM, Matthews PM (2004) Advances in functional and structural MR image analysis and implementation as FSL. Neuroimage 23:S208-S219. CrossRef Medline

Stüber C, Morawski M, Schäfer A, Labadie C, Wähnert M, Leuze C, Streicher M, Barapatre N, Reimann K, Geyer S, Spemann D, Turner R (2014) Myelin and iron concentration in the human brain: a quantitative study of MRI contrast. Neuroimage 93:95-106. CrossRef Medline

Sutter ML, Schreiner CE, McLean M, O'Connor KN, Loftus WC (1999) Organization of inhibitory frequency receptive fields in cat primary auditory cortex. J Neurophysiol 82:2358-2371. Medline

Sweet RA, Dorph-Petersen KA, Lewis DA (2005) Mapping auditory core, lateral belt, and parabelt cortices in the human superior temporal gyrus. J Comp Neurol 491:270-289. CrossRef Medline

Talavage TM, Edmister WB (2004) Nonlinearity of FMRI responses in human auditory cortex. Hum Brain Mapp 22:216-228. CrossRef Medline

Talavage TM, Sereno MI, Melcher JR, Ledden PJ, Rosen BR, Dale AM (2004) Tonotopic organization in human auditory cortex revealed by progressions of frequency sensitivity. J Neurophysiol 91:1282-1296. Medline

Tardif CL, Schäfer A, Waehnert M, Dinse J, Turner R, Bazin PL (2015) Multi-contrast multi-scale surface registration for improved alignment of cortical areas. Neuroimage 111:107-122. CrossRef Medline

Tardif CL, Gauthier CJ, Steele CJ, Bazin PL, Schäfer A, Schaefer A, Turner R, Villringer A (2016) Advanced MRI techniques to improve our understanding of experience-induced neuroplasticity. Neuroimage 131:55-72. CrossRef Medline

Thomas JM, Huber E, Stecker GC, Boynton GM, Saenz M, Fine I (2015) Population receptive field estimates of human auditory cortex. Neuroimage 105:428-439. CrossRef Medline

Turner R (2015) Myelin imaging. In: Brain mapping: an encyclopedic reference, pp 137-142. San Diego: Academic Elsevier.

Wallace MN, Cronin MJ, Bowtell RW, Scott IS, Palmer AR, Gowland PA (2016) Histological basis of laminar MRI patterns in high resolution images of fixed human auditory cortex. Front Neurosci 10:455. CrossRef Medline

Wehr M, Zador AM (2003) Balanced inhibition underlies tuning and sharpens spike timing in auditory cortex. Nature 426:442-446. CrossRef Medline

Woods DL, Stecker GC, Rinne T, Herron TJ, Cate AD, Yund EW, Liao I, Kang X (2009) Functional maps of human auditory cortex: effects of acoustic features and attention. PLoS One 4:e5183. CrossRef Medline 